TRANSACTIONS OF THE

AMERICAN MATHEMATICAL SOCIETY

Volume 361, Number 1, January 2009, Pages 297-329

S 0002-9947(08)04480-2

Article electronically published on August 14, 2008

\title{
CRYSTAL BASES AND COMBINATORICS OF INFINITE RANK QUANTUM GROUPS
}

\author{
CÉDRIC LECOUVEY
}

\begin{abstract}
The tensor powers of the vector representation associated to an infinite rank quantum group decompose into irreducible components with multiplicities independent of the infinite root system considered. Although the irreducible modules obtained in this way are not of highest weight, they admit a crystal basis and a canonical basis. This permits us in particular to obtain for each family of classical Lie algebras a Robinson-Schensted correspondence on biwords defined on infinite alphabets. We then depict a structure of bicrystal on these biwords. This RSK-correspondence yields also a plactic algebra and plactic Schur functions distinct for each infinite root system. Surprisingly, the algebras spanned by these plactic Schur functions are all isomorphic to the algebra of symmetric functions.
\end{abstract}

\section{INTRODUCTION}

The diagonal expansion of the Cauchy kernel as a sum over all partitions $\lambda$

$$
\prod_{i \in I} \prod_{j \in J}\left(1-x_{i} y_{j}\right)^{-1}=\sum_{\lambda} s_{\lambda}(X) s_{\lambda}(Y)
$$

permits one to characterize the Schur functions $s_{\lambda}$ (see [14]). This Cauchy identity can be interpreted in terms of the combinatorics of Young tableaux by using the Robinson-Schensted-Knuth correspondence, that is, the Robinson-Schensted correspondence on biwords defined on the totally ordered alphabets $\mathcal{X}=\left\{x_{i} \mid i \in I\right\}$ and $\mathcal{Y}=\left\{y_{j} \mid j \in J\right\}$ (see [3] ). Recall that a biword $W$ on $\mathcal{X}$ and $\mathcal{Y}$ can be written

$$
W=\left(\begin{array}{ccccccccccccccc}
y_{1} & \cdot & \cdot & y_{1} & y_{2} & \cdot & \cdot & y_{2} & \cdot & \cdot & \cdot & y_{k} & \cdot & \cdot & y_{k} \\
b_{1} & \cdot & \cdot & e_{1} & b_{2} & \cdot & \cdot & e_{2} & \cdot & \cdot & \cdot & b_{k} & \cdot & \cdot & e_{k}
\end{array}\right)=\left(\begin{array}{c}
w_{y} \\
w_{x}
\end{array}\right),
$$

where for any $p \in\{1, \ldots, k\}, b_{p} \cdots e_{p}$ is a word on $\mathcal{X}$ such that $b_{p} \geq \cdots \geq e_{p}$. The RSK-correspondence associates to each biword $W \in \mathcal{W}$ a pair of semistandard tableaux $(P, Q)$ with the same shape where $P$ is obtained by applying the Schensted bumping algorithm to $w_{x} \in \mathcal{X}$ and $Q$ is the corresponding recording tableau with evaluation $w_{y}$. By identifying each word $w$ of length $\ell$ on $\mathcal{X}$ with the biword $\left(\begin{array}{c}12 \cdots l \\ w\end{array}\right)$, one also obtains a correspondence between words $w$ on $\mathcal{X}$ and pairs $(P(w), Q(w))$ where $Q(w)$ is a standard tableau. This makes naturally appear the plactic monoid defined as the quotient of the free monoid on $\mathcal{X}$ by the Knuth relations

$$
\left\{\begin{array}{l}
a b x=b a x \text { if } a<x \leq b \\
a b x=a x b \text { if } x \leq a<b
\end{array}\right.
$$

Received by the editors May 12, 2006 and, in revised form, December 30, 2006, January 10, 2007, and January 17, 2007.

2000 Mathematics Subject Classification. Primary 05-02, 17B10, 17B37, $17 \mathrm{~B} 67$.

(C)2008 American Mathematical Society 297

Reverts to public domain 28 years from publication 
More precisely, two words $w_{1}$ and $w_{2}$ of length $\ell$ on $\mathcal{X}$ are congruent in the plactic monoid if and only if $P\left(w_{1}\right)=P\left(w_{2}\right)$. Thanks to this monoid, it is possible to embed the algebra of symmetric functions into a noncommutative algebra called the plactic algebra which permits one to obtain an illuminating proof of the Littlewood-Richardson rule 20. The plactic monoid also naturally occurs in the theory of crystal bases developed by Kashiwara [11. To each finite dimensional $U_{q}\left(\mathfrak{s l}_{n}\right)$-module $M$ is associated a crystal graph whose decomposition in connected components reflects the decomposition of $M$ into its irreducible components. Let $B^{\otimes \ell}$ be the crystal graph of the $\ell$-th tensor power of the vector representation (i.e. the fundamental $n$-dimensional $U_{q}\left(\mathfrak{s l}_{n}\right)$-module). The vertices of $B^{\otimes \ell}$ can be identified with the words of length $\ell$ on $\mathcal{X}$. The plactic monoid is then obtained by identifying the vertices of $B^{\otimes \ell}$ which occur at the same place in two isomorphic connected components. Similarly, the RSK correspondence can be interpreted as an isomorphism of crystal graphs (see 95.31$)$. Moreover it naturally endows $\mathcal{W}$ with the structure of a bi-crystal graph ([2], [14]).

In the early 1990s, Robinson-Schensted type correspondences were also obtained for the other classical Lie algebras. This was notably realized by Berele in [1 for $\mathfrak{s p}_{2 n}$, by Okada in 21] for $\mathfrak{s o}_{2 n}$ and by Sundaram in 24, for $\mathfrak{s o}_{2 n+1}$ (see also [6] for a complete list of references). Nevertheless, it seems difficult to derive plactic relations from these schemes and to relate them to the Kashiwara crystal basis theory. In fact, crystal basis theory permits us to define plactic monoids and to obtain RSK-type correspondences on words for the quantum groups $U_{q}\left(\mathfrak{s o}_{2 n+1}\right), U_{q}\left(\mathfrak{s p}_{2 n}\right)$ and $U_{q}\left(\mathfrak{s o}_{2 n}\right)$ in a natural way ([15, [16]). These correspondences are based on insertion algorithms for tableaux of types $B_{n}, C_{n}$ and $D_{n}[12$ which extend the notion of semistandard tableaux to the other classical types. Note that these tableaux, whose combinatorial definition is deduced from the action of the Kashiwara crystal operators, do not coincide with those used by Berele, Okada and Sundaram. Thus the correspondences obtained from crystal graph theory do not coincide with those described in [1], 21] and 24]. For each type $\Delta_{n}, \Delta=B, C, D$, one can also consider biwords of type $\Delta_{n}$ and associate to each such biword $W$ a pair $\left(P_{\Delta_{n}}, Q_{\Delta_{n}}\right)$ where $P_{\Delta_{n}}$ is a tableau of type $\Delta_{n}$ and $Q_{\Delta_{n}}$ a recording tableau. This recording tableau consists of a sequence of Young diagrams $Q_{1}, \ldots, Q_{\ell}$ where the number of boxes in $Q_{i}$ can be strictly greater than that of $Q_{i+1}$. This implies in particular that the association $W \mapsto\left(P_{\Delta_{n}}, Q_{\Delta_{n}}\right)$ cannot yield a one-to-one correspondence on biwords of type $\Delta_{n}$. Nevertheless, we are going to see that it is possible to overcome this problem by considering crystals and biwords associated to infinite rank quantum groups. Note that, when the association $W \mapsto\left(P_{\Delta_{n}}, Q_{\Delta_{n}}\right)$ is restricted to words $w$ via the identification $w=\left(\begin{array}{c}12 \cdots l \\ w\end{array}\right)$, the sequence of Young diagrams $Q_{1}, \ldots, Q_{\ell}$ obtained is such that $Q_{i}$ and $Q_{i+1}$ differ by at most one box. In this special case, it is established in [15] and [16] that the map $w \rightarrow\left(P_{\Delta_{n}}, Q_{\Delta_{n}}\right)$ is a one-to-one correspondence; thus the use of infinite rank crystals is not required.

Let $\mathfrak{g}_{\infty}$ be a Lie algebra of type $\Delta_{\infty}=A_{\infty}, B_{\infty}, C_{\infty}$ or $D_{\infty}$. In this paper, we show that the combinatorial constructions and properties related to the root systems of type $A_{n}, n \geq 1$ we have described above can be generalized to the infinite root systems $\Delta_{\infty}$. More precisely, we show that for each infinite root system $\Delta_{\infty}$, there exists an RSK-type correspondence (thus bijective) $W \mapsto\left(P_{\infty}, Q_{\infty}\right)$ on biwords defined on two ordered alphabets $\mathcal{X}$ and $\mathcal{Y}$ where $P_{\infty}$ and $Q_{\infty}$ are tableaux respectively of type $\Delta_{\infty}$ and semi-standard with the same shape. Contrary to the 
correspondences previously obtained by Berele, Okada and Sundaram, our RSKcorrespondences yield the structure of a bi-crystal (that is, for $U_{q}\left(\mathfrak{g}_{\infty}\right)$ and $U_{q}\left(\mathfrak{s l}_{\infty}\right)$ ) on biwords answering in particular to a problem evoked in [25. The combinatorial description of these RSK-type correspondences is based on the existence of a plactic monoid for each type $\Delta_{\infty}$. The defining relations of these monoids depend on the root system considered. They permit us to introduce a plactic algebra and plactic Schur functions of type $\Delta_{\infty}$. We show that the plactic Schur functions of type $\Delta_{\infty}$ span a commutative algebra isomorphic to the algebra of symmetric functions. In particular the four algebras obtained in this way are isomorphic. Our correspondence on biwords also gives Cauchy-type identities on the alphabets $\mathcal{X}$ and $\mathcal{Y}$ analogous to (11) for types $B_{\infty}, C_{\infty}$ and $D_{\infty}$. These formulas hold only in the infinite rank case and do not permit us to recover Littlewood's formulas described in [19] (see Remark after (33)). Note that a combinatorial proof of Littlewood's identity for type $C_{n}$ has been obtained by Sundaram (see [23]), but the combinatorics they require is not, as far as the author is aware, compatible with crystal basis theory.

To establish our combinatorial results, we need an extension of the crystal basis theory to certain irreducible $U_{q}\left(\mathfrak{g}_{\infty}\right)$-modules $M_{\infty}$ which are not of highest weight. These modules are the irreducible components which appear in the decomposition of the tensor powers of the vector representations associated to the quantum groups of infinite rank. They are irreducible $U_{q}\left(\mathfrak{g}_{\infty}\right)$-modules $V_{\infty}(\lambda)$ naturally labelled by partitions. Since we have not found references on these modules in the literature, we have devoted two sections of the paper to the exposition of their algebraic properties. We establish notably the decomposition

$$
V_{\infty}(\lambda) \otimes V_{\infty}(\lambda) \simeq \bigoplus_{\nu} V_{\nu}(\lambda)^{\oplus c_{\lambda, \mu}^{\nu}}
$$

where $c_{\lambda, \mu}^{\nu}$ is the Littlewood-Richardson coefficient associated to $\lambda, \mu$ and $\nu$. Note that the decomposition (2) is independent of the infinite root system considered. We also show how to define crystal bases for the modules $M_{\infty}$ so that the irreducible modules $V_{\infty}(\lambda)$ have a unique crystal basis (up to an overall renormalization) and a canonical basis. The general philosophy of the paper is to introduce the algebraic and combinatorial objects related to the infinite rank quantum groups as relevant direct limits of their analogues in finite rank.

The paper is organized as follows. In section 2, we review the necessary background on quantum infinite rank Lie algebras and recall the crystal basis theory for their irreducible highest weight modules $\mathcal{V}(\lambda)$, which is very close to that corresponding to the finite rank case but leads to infinite crystal graphs. Although these modules $\mathcal{V}(\lambda)$ are not directly needed for our RSK-type correspondence, we have thought that a combinatorial description of their crystals could be useful, especially to avoid a possible confusion with infinite crystals used in Section 4. Section 3 is devoted to the definition of the modules $V_{\infty}(\lambda)$ and the proof of the LittlewoodRichardson rule (2). We introduce the crystal bases of the modules $M_{\infty}$ and the canonical bases of the modules $V_{\infty}(\lambda)$ in Section 4. The RSK-type correspondence and the bi-crystal structures are described in Section 5. Finally we prove in Section 6 that the algebras spanned by the plactic Schur functions are all isomorphic to the algebra of symmetric functions and establish Cauchy-type identities. 


\section{BACKGROUND}

In this section we introduce the infinite rank Lie algebras we consider in this paper together with the corresponding quantum groups. We also include a few considerations on their highest weight modules to avoid a possible confusion with the modules $V_{\infty}(\lambda)$ (which are not of highest weight) of Section 3 . The reader is referred to [4, [7, 8], 10] and [1] for more details.

2.1. Quantum groups of infinite rank. Let $\mathfrak{g l}_{ \pm \infty}$ be the Lie algebra of complex $\mathbb{Z} \times \mathbb{Z}$ matrices $A=\left(a_{i, j}\right)$ with a finite number of nonzero entries. The Lie algebra $\mathfrak{g l}_{ \pm \infty}$ contains Lie subalgebras $\mathfrak{g}_{A_{\infty}}, \mathfrak{g}_{B_{\infty}}, \mathfrak{g}_{C_{\infty}}$ and $\mathfrak{g}_{D_{\infty}}$ of types $A_{\infty}, B_{\infty}, C_{\infty}$ and $D_{\infty}$ (see [9], $\left.\S 7.11\right)$. To make our notation homogeneous we write $A_{\infty}$ for the infinite Dynkin diagram which is denoted by $A_{+\infty}$ in $[9]$ :

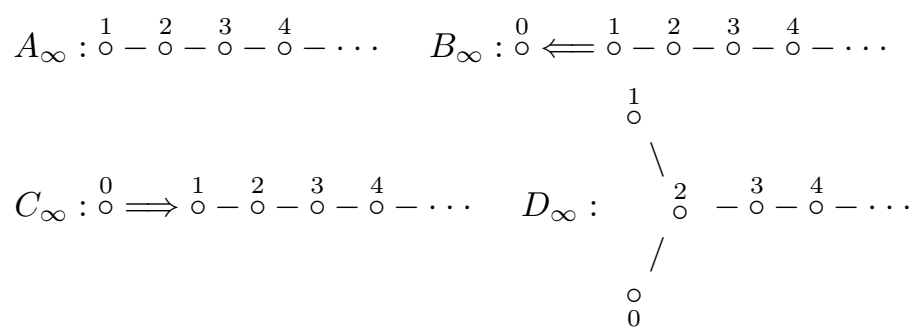

Note that the infinite rank Lie algebras we use in this paper can all be realized as Lie algebras of infinite matrices with a finite number of nonzero entries. The nodes of the Dynkin diagrams (3) are labelled by $I$, where $I=\mathbb{N}-\{0\}$ for type $A_{\infty}$ and $I=\mathbb{N}$ for types $B_{\infty}, C_{\infty}$ and $D_{\infty}$.

We denote by $e_{i}, f_{i}, t_{i}, i \in I$, the set of Chevalley generators of $U_{q}\left(\mathfrak{g}_{\infty}\right)$. According to our convention for the labelling of the infinite Dynkin diagrams (3), the fundamental weights of $U_{q}\left(\mathfrak{g}_{\infty}\right)$ belong to $\left(\frac{\mathbb{Z}}{2}\right)^{\infty}$. More precisely we have $\omega_{i}=\left(0^{i}, 1,1, \ldots\right)$ for $i \geq 2$ and also $i=1$ for $\mathfrak{g}_{\infty} \neq \mathfrak{g}_{D_{\infty}}, \omega_{0}^{C_{\infty}}=(1,1,1, \ldots), \omega_{0}^{B}=\omega_{0}^{D_{\infty}}=\left(\frac{1}{2}, \frac{1}{2}, \ldots\right)$ and $\omega_{1}^{D \infty}=\left(-\frac{1}{2}, \frac{1}{2}, \ldots\right)$. The weight lattice $P$ of $U_{q}\left(\mathfrak{g}_{\infty}\right)$ can be considered as the $\mathbb{Z}$-sublattice of $\left(\frac{\mathbb{Z}}{2}\right)^{\infty}$ generated by the $\omega_{i}, i \in I$. So a weight $\delta$ associated to $U_{q}\left(\mathfrak{g}_{\infty}\right)$ is an infinite sequence $\delta=\left(\delta_{1}, \delta_{2}, \ldots\right)$ of integers (resp. half integers) for $\mathfrak{g}_{\infty}=\mathfrak{g}_{A_{\infty}}, \mathfrak{g}_{C_{\infty}}$ (resp. $\left.\mathfrak{g}_{\infty}=\mathfrak{g}_{B}, \mathfrak{g}_{D_{\infty}}\right)$. We denote by $P^{+}$the cone of dominant weights of $U_{q}\left(\mathfrak{g}_{\infty}\right)$, that is, the set of finite linear combinations of fundamental weights with nonnegative integer coefficients. They correspond to the infinite sequences $\lambda=\left(\lambda_{1}, \lambda_{2}, \ldots\right) \in P$ such that

$$
0 \leq \lambda_{1} \leq \lambda_{2} \leq \lambda_{3} \leq \cdots \leq \lambda_{i}=\lambda_{i+1}=\lambda_{i+2}=\cdots
$$

except for type $D_{\infty}$ in which case the inequality $\lambda_{1} \leq \lambda_{2}$ above is replaced by $\left|\lambda_{1}\right| \leq \lambda_{2}$.

Let $\varepsilon_{i}$ be the $i$-th standard basis vector of $\mathbb{Z}^{\infty}$. The simple roots $\left\{\alpha_{i} \mid i \in I\right\}$ are given by $\alpha_{i}=\varepsilon_{i+1}-\varepsilon_{i}$ for $i \neq 0, \alpha_{0}^{B}=-\varepsilon_{1}, \alpha_{0}^{C_{\infty}}=-2 \varepsilon_{1}$ and $\alpha_{0}^{D_{\infty}}=-\varepsilon_{1}-\varepsilon_{2}$. Write $Q$ for the root lattice, that is, for the $\mathbb{Z}$-sublattice of $\mathbb{Z}^{\infty}$ generated by the simple roots and set $Q^{+}=Q \cap P^{+}$. Given $\delta$ and $\gamma$ two weights in $P$, we will write $\delta \leq \gamma$ when $\delta-\gamma$ belongs to $Q_{+}$.

For any nonnegative integer $n$ set $I_{n}=\{i \in I \mid i<n\}$ and denote by $U_{q}\left(\mathfrak{g}_{n}\right)$ the subalgebra of $U_{q}\left(\mathfrak{g}_{\infty}\right)$ generated by $e_{i}, f_{i}, t_{i}, i \in I_{n}$. Clearly $U_{q}\left(\mathfrak{g}_{n}\right)$ is the quantum group associated to the finite root system obtained by considering only the simple roots labelled by $i \in I_{n}$ in (3). For any $\delta=\left(\delta_{1}, \delta_{2}, \ldots\right) \in P$, set 
$\pi_{n}(\delta)=\left(\delta_{1}, \delta_{2}, \ldots, \delta_{n}\right)$. Denote by $P_{n}$ and $Q_{n}$ the weight and root lattices of $U_{q}\left(\mathfrak{g}_{n}\right)$. One has $P_{n}=\pi_{n}(P)$ and $Q_{n}=\pi_{n}(Q)$. Moreover the set of dominant weights for $U_{q}\left(\mathfrak{g}_{n}\right)$ verifies $P_{n}^{+}=\pi_{n}\left(P^{+}\right)$. For any integer $p \geq n$, it will be convenient to identify $P_{n}$ with the sublattice of $P_{p}$ containing weights of the form $\left(\delta_{1}, \ldots, \delta_{n}, 0, \ldots, 0\right)$. Note that the dominant weights of $P_{n}^{+}$are not dominant in $P_{p}$ via this identification. For any $\delta=\left(\delta_{1}, \ldots, \delta_{n}\right) \in P_{n}$, we set $|\delta|=\delta_{1}+\cdots+\delta_{n}$.

Given two $U_{q}\left(\mathfrak{g}_{\infty}\right)$-modules $M$ and $N$, the structure of a $U_{q}\left(\mathfrak{g}_{\infty}\right)$-module on $M \otimes N$ is defined by putting

$$
\begin{gathered}
q^{h}(u \otimes v)=q^{h} u \otimes q^{h} v \\
e_{i}(u \otimes v)=e_{i} u \otimes t_{i}^{-1} v+u \otimes e_{i} v, \\
f_{i}(u \otimes v)=f_{i} u \otimes v+t_{i} u \otimes f_{i} v
\end{gathered}
$$

for any $i \in I$.

2.2. Modules of the category $\mathcal{O}_{\text {int }}$. The reader is referred to 8 for a complete exposition of the results on the category $\mathcal{O}_{\text {int }}$ in the finite rank case. We also denote by $\mathcal{O}_{\text {int }}$ the category of $U_{q}\left(\mathfrak{g}_{\infty}\right)$-modules $\mathcal{M}$ satisfying the following conditions:

(1) $\mathcal{M}$ has a weight space decomposition; that is, $\mathcal{M}=\bigoplus_{\delta \in P} \mathcal{M}_{\delta}$, where $\mathcal{M}_{\delta}=\left\{v \in \mathcal{M} \mid q^{h_{i}} v=q^{\delta\left(h_{i}\right)}\right.$ for all $\left.i \in \mathbb{N}\right\}$ and $\operatorname{dim} \mathcal{M}_{\delta}<\infty$.

(2) There exists a finite number of weights $\lambda^{(1)}, \lambda^{(2)}, \ldots, \lambda^{(s)}$ such that

$$
\mathcal{M}_{\delta} \neq\{0\} \Longrightarrow \delta \in D\left(\lambda^{(1)}\right) \cup \cdots \cup D\left(\lambda^{(s)}\right),
$$

where for any $k=1, \ldots, s, D\left(\lambda^{(k)}\right)=\left\{\gamma \in P \mid \gamma \leq \lambda^{(k)}\right\}$.

(3) The generators $e_{i}, f_{i}, i \in I_{n}$ are locally nilpotent on $\mathcal{M}$.

By using arguments similar to those exposed in $\left[8\right.$, we obtain that the $U_{q}\left(\mathfrak{g}_{\infty}\right)$ modules of the category $\mathcal{O}_{\text {int }}$ are totally reducible and admit crystal bases. If $(L, B)$ and $\left(L^{\prime}, B^{\prime}\right)$ are crystal bases of the $U_{q}\left(\mathfrak{g}_{\infty}\right)$-modules $\mathcal{M}$ and $\mathcal{M}^{\prime}$ belonging to $\mathcal{O}_{\text {int }}$, then $\left(L \otimes L^{\prime}, B \otimes B^{\prime}\right)$ with $B \otimes B^{\prime}=\left\{b \otimes b^{\prime} ; b \in B, b^{\prime} \in B^{\prime}\right\}$ is a crystal basis of $\mathcal{M} \otimes \mathcal{M}^{\prime}$. The action of the Kashiwara operators $\widetilde{e}_{i}$ and $\widetilde{f}_{i}$ on $B \otimes B^{\prime}$ is given by

$$
\widetilde{f}_{i}(u \otimes v)=\left\{\begin{array}{l}
\tilde{f}_{i}(u) \otimes v \text { if } \varphi_{i}(u)>\varepsilon_{i}(v), \\
u \otimes \widetilde{f}_{i}(v) \text { if } \varphi_{i}(u) \leq \varepsilon_{i}(v),
\end{array}\right.
$$

and

$$
\widetilde{e_{i}}(u \otimes v)=\left\{\begin{array}{l}
u \otimes \widetilde{e_{i}}(v) \text { if } \varphi_{i}(u)<\varepsilon_{i}(v), \\
\widetilde{e_{i}}(u) \otimes v \text { if } \varphi_{i}(u) \geq \varepsilon_{i}(v),
\end{array}\right.
$$

where $\varepsilon_{i}(u)=\max \left\{k ; \widetilde{e}_{i}^{k}(u) \neq 0\right\}$ and $\varphi_{i}(u)=\max \left\{k ; \widetilde{f}_{i}^{k}(u) \neq 0\right\}$.

The set $B$ may be endowed with a combinatorial structure called the crystal graph of $\mathcal{M}$. Crystal graphs for $U_{q}\left(\mathfrak{g}_{\infty}\right)$-modules in $\mathcal{O}_{\text {int }}$ are infinite oriented colored graphs with colors $i \in I$. An arrow $a \stackrel{i}{\rightarrow} b$ means that $\tilde{f}_{i}(a)=b$ and $\widetilde{e}_{i}(b)=$ $a$. The decomposition of $\mathcal{M}$ into its irreducible components is reflected into the decomposition of $B$ into its connected components.

The weight of $b \in B$ is denoted $\operatorname{wt}(b)$ and we have

$$
\operatorname{wt}(b)=\sum_{i \in I}\left(\varphi_{i}(b)-\varepsilon_{i}(b)\right) \omega_{i} \in P .
$$

The following lemma is a straightforward consequence of (7) and (8). 
Lemma 2.2.1. Let $u \otimes v \in B \otimes B^{\prime}$. Then, $u \otimes v$ is a highest weight vertex of $B \otimes B^{\prime}$ if and only if for any $i \in I, \widetilde{e}_{i}(u)=0$ (i.e. $u$ is of highest weight) and $\varepsilon_{i}(v) \leq \varphi_{i}(u)$.

2.3. The highest weight modules $\mathcal{V}(\lambda)$. For any dominant weight $\lambda \in P^{+}$, we denote by $\mathcal{V}(\lambda)$ the irreducible highest weight $U_{q}\left(\mathfrak{g}_{\infty}\right)$-module of highest weight $\lambda$. It is an infinite dimensional module which belongs to the category $\mathcal{O}_{\text {int }}$. Thus $\mathcal{V}(\lambda)$ admits a crystal basis $(L(\lambda), B(\lambda))$ unique up to an overall scalar factor. More precisely there exists a highest weight vector $v_{\lambda}$ in $\mathcal{V}(\lambda)$ such that

$$
\begin{aligned}
L(\lambda) & =\bigoplus_{r \geq 0, i_{k} \in I} \mathbf{A}(q) \widetilde{f}_{i_{1}} \cdots \widetilde{f}_{i_{r}} v_{\lambda}, \\
B(\lambda) & =\left\{\widetilde{f}_{i_{1}} \cdots \widetilde{f}_{i_{r}} v_{\lambda}+q L(\lambda) \in L(\lambda) / q L(\lambda) \mid r \geq 0, i_{k} \in I\right\}-\{0\},
\end{aligned}
$$

where $\mathbf{A}(q)$ is the subalgebra of $\mathbb{Q}(q)$ consisting of the rational functions without pole at $q=0$ and the $\widetilde{f}_{i_{k}}$ 's are the Kashiwara operators.

Lemma 2.3.1. Consider $v_{\lambda}$ and $v_{\lambda}^{\prime}$ two highest weight vectors in $\mathcal{V}(\lambda)$ and denote respectively by $(L(\lambda), B(\lambda))$ and $\left(L^{\prime}(\lambda), B^{\prime}(\lambda)\right)$ the crystal bases obtained from $v_{\lambda}$ and $v_{\lambda}^{\prime}$ as in (10). Then $(L(\lambda), B(\lambda))=\left(L^{\prime}(\lambda), B^{\prime}(\lambda)\right)$ if and only if there exists $K \in \mathbf{A}(q)$ with $K(0)=1$ such that $v_{\lambda}^{\prime}=K(q) v_{\lambda}$.

Proof. Suppose that $(L(\lambda), B(\lambda))=\left(L^{\prime}(\lambda), B^{\prime}(\lambda)\right)$. Since $L(\lambda)=L^{\prime}(\lambda)$ we must have by (10) $v_{\lambda}^{\prime} \in L(\lambda)$. Thus there exists $K(q) \in \mathbf{A}(q)$ such that $v_{\lambda}^{\prime}=K(q) v_{\lambda}$. Moreover $v_{\lambda}^{\prime}+q L(\lambda) \in B(\lambda)$ for $B^{\prime}(\lambda)=B(\lambda)$. Since $v_{\lambda}+q L(\lambda)$ is the unique vertex in $B(\lambda)$ of weight $\lambda$, we obtain $v_{\lambda}^{\prime}+q L(\lambda)=v_{\lambda}+q L(\lambda)$. By using the equality $v_{\lambda}^{\prime}=K(q) v_{\lambda}$, we derive $K(0)=1$.

Conversely, if $v_{\lambda}^{\prime}=K(q) v_{\lambda}$ with $K(0)=1$, we have $L(\lambda)=L^{\prime}(\lambda)$ by (10) because $K(q)$ is invertible in $\mathbf{A}(q)$. Moreover, $v_{\lambda}^{\prime}+q L(\lambda)=v_{\lambda}+q L(\lambda)$ for $v_{\lambda}^{\prime}=$ $v_{\lambda}+(K(q)-1) v_{\lambda}$ with $K(q)-1 \in q L(\lambda)$. Thus $B^{\prime}(\lambda)=B(\lambda)$.

For any positive integer $n$, set $V_{n}(\lambda)=U_{q}\left(\mathfrak{g}_{n}\right) \cdot v_{\lambda}$. Then $V_{n}(\lambda)$ is a finite dimensional $U_{q}\left(\mathfrak{g}_{n}\right)$-module of highest weight $\pi_{n}(\lambda)$ and highest vector $v_{\lambda}$. We denote by $\left(L_{n}(\lambda), B_{n}(\lambda)\right)$ the crystal basis of $V_{n}(\lambda)$ obtained as in (10) by authorizing only indices $i_{k} \in I_{n}$. Clearly, the previous lemma also holds for the finite dimensional highest weight $U_{q}\left(\mathfrak{g}_{n}\right)$-modules $V_{n}(\lambda)$. Since $L_{n}(\lambda)=L(\lambda) \cap V_{n}(\lambda)$, the map

$$
\kappa_{n}:\left\{\begin{array}{l}
B_{n}(\lambda) \rightarrow B(\lambda) \\
\widetilde{f}_{i_{1}} \cdots \widetilde{f}_{i_{r}} v_{\lambda}+q L_{n}(\lambda) \mapsto \widetilde{f}_{i_{1}} \cdots \widetilde{f}_{i_{r}} v_{\lambda}+q L(\lambda)
\end{array}\right.
$$

is an embedding of crystals. The crystal $B_{n}(\lambda)$ is then isomorphic to the subcrystal of $B(\lambda)$ obtained by considering the vertices which are connected to the highest weight vertex $b_{\lambda}=v_{\lambda}+q L(\lambda)$ by a path colored with colors $i \in I_{n}$. In [12, Kashiwara and Nakashima have obtained a natural labelling of the crystal $B_{n}(\lambda)$ by tableaux of shape $\pi_{n}(\lambda)$. The definition of these tableaux depends on the root system considered (see Section 5). For $A_{n}$ they coincide with the well-known semistandard tableaux. This labelling of $B_{n}(\lambda)$ induces a natural labelling of $B(\lambda)$. Indeed consider a vertex $b \in B(\lambda)$ and let $m$ be the minimal integer such that $b$ belongs to the image of $\kappa_{m}$. Then one labels the vertex $b$ by the unique tableau $T$ such that $\kappa_{m}(T)=b$. The weight of $b$ is then equal to $\operatorname{wt}(b)=\left(d_{1}, \ldots, d_{m}, \lambda_{m+1}, \lambda_{m+2}, \ldots\right)$ where for any $i \in\{1, \ldots, m\}, d_{i}$ is the number of letters $\bar{i}$ minus the number of letters $i$ in $T$. This means that the vertex labelled by $T$ must in fact be thought of as the infinite tableau obtained by adding on the top of $T$, first a row of length 
$\lambda_{m+1}$ containing letters $\overline{m+1}$, next a row of length $\lambda_{m+2}$ containing letters $\overline{m+2}$, and so on. For any positive integer $n$, the crystal $B_{n}(\lambda)$ is obtained by deleting simultaneously in $B(\lambda)$ all the arrows colored by $i \geq n$ together with the vertices they connect. Note that each vertex $b \in B_{n}(\lambda) \subset B(\lambda)$ is such that $\widetilde{f}_{n}(b) \neq 0$.

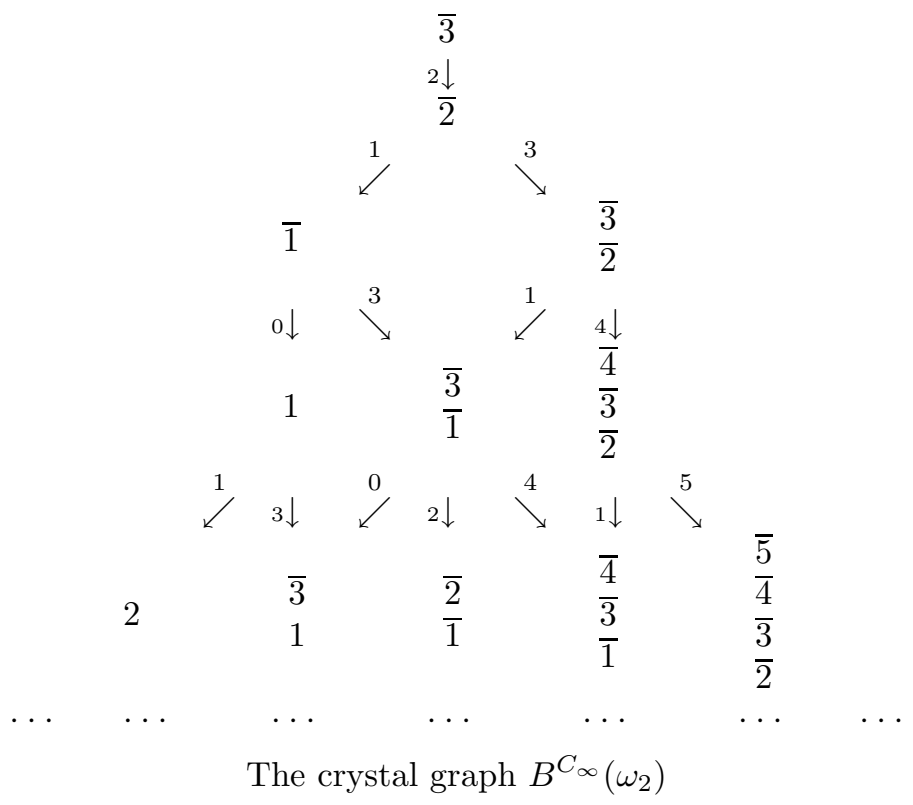

Note that our conventions for labelling the crystal graph of the vector representations are not those used by Kashiwara and Nakashima 12. To obtain the original description of $B_{n}(\lambda)$ from that used in the sequel it suffices to change each letter $k \in\{1, \ldots, n\}$ into $\overline{n-k+1}$ and each letter $\bar{k} \in\{\overline{1}, \ldots, \bar{n}\}$ into $n-k+1$.

Consider $\lambda$ and $\mu$ two dominant weights of $P^{+}$. For any positive integer $n$ set

$$
\begin{array}{r}
E_{n}(\lambda, \mu)=\left\{\nu \in P^{+} \mid \nu=\left(\nu_{1}, \ldots, \nu_{n}, \lambda_{n+1}+\mu_{n+1}, \lambda_{n+2}+\mu_{n+2}, \ldots\right)\right. \\
\text { with } \left.\pi_{n}(\nu) \in P_{n}^{+} \text {and } \nu_{n} \neq \lambda_{n}+\mu_{n}\right\} .
\end{array}
$$

Proposition 2.3.2. With the above notation we have

$$
\mathcal{V}(\lambda) \otimes \mathcal{V}(\mu) \simeq \bigoplus_{n \geq 1} \bigoplus_{\nu \in E_{n}(\lambda, \mu)} \mathcal{V}(\nu)^{\oplus m_{\lambda, \mu}^{n, \nu},}
$$

where $m_{\lambda, \mu}^{n, \nu}$ is the multiplicity of $V_{n}(\nu)$ in the tensor product $V_{n}(\lambda) \otimes V_{n}(\mu)$.

Proof. Suppose that $b=b_{1} \otimes b_{2} \in B(\lambda) \otimes B(\mu)$ is of highest weight $\nu$. Then we must have $\widetilde{e}_{i}(b)=0$ for any $i \in I$. Thus it follows by Lemma 2.2 .1 that $b_{1}$ is the highest weight vertex of $B(\lambda)$ (thus has weight $\lambda$ ) and $\varepsilon_{i}\left(b_{2}\right) \leq \lambda_{i+1}-\lambda_{i}$ for any $i \in I$. Since $b_{2} \in B(\mu)$ there exists a nonnegative integer $n$ such that $\operatorname{wt}\left(b_{2}\right)=\left(\delta_{1}, \ldots, \delta_{n}, \mu_{n+1}, \mu_{n+2}, \ldots\right)$ and $\delta_{n} \neq \mu_{n}$. Then we must have

$$
\nu=\operatorname{wt}\left(b_{1}\right)+\operatorname{wt}\left(b_{2}\right)=\left(\lambda_{1}+\delta_{1}, \ldots, \lambda_{n}+\delta_{n}, \lambda_{n+1}+\mu_{n+1}, \lambda_{n+2}+\mu_{n+2}, \ldots\right) .
$$

For any $i \in\{1, \ldots, n-1\}$ we have $\left(\lambda_{i+1}+\delta_{i+1}\right)-\left(\lambda_{i}+\delta_{i}\right) \geq \varepsilon_{i}\left(b_{2}\right)+\delta_{i+1}-\delta_{i}$. Since $\delta_{i+1}-\delta_{i}=\varphi_{i}\left(b_{2}\right)-\varepsilon_{i}\left(b_{2}\right)$, we obtain $\left(\lambda_{i+1}+\delta_{i+1}\right)-\left(\lambda_{i}+\delta_{i}\right) \geq 0$. Thus $\nu \in$ $E_{n}(\lambda, \mu)$ and $\kappa_{n}^{-1}(b)$ is a highest weight vertex of weight $\pi_{n}(\lambda)$ in $V_{n}(\lambda) \otimes V_{n}(\mu)$. 
This means that $V_{n}(\lambda) \otimes V_{n}(\mu)$ contains an irreducible component isomorphic to $V_{n}(\nu)$.

Conversely, consider $\nu \in E_{n}(\lambda, \mu)$ such that $V_{n}(\lambda) \otimes V_{n}(\mu)$ contains an irreducible component isomorphic to $V_{n}(\nu)$. Let $b^{(n)}$ be the highest weight vertex of $V_{n}(\lambda) \otimes$ $V_{n}(\mu)$ associated to this component. Then $b=\kappa_{n}\left(b^{(n)}\right)$ is a highest weight vertex of weight $\nu$ in $B(\lambda) \otimes B(\mu)$.

Example 2.3.3. The square of the spin representation $\mathcal{V}\left(\omega_{0}\right)$ of $U_{q}\left(\mathfrak{g}_{B_{\infty}}\right)$ is isomorphic to the direct sum

$$
\mathcal{V}\left(\omega_{0}\right)^{\otimes 2} \simeq \mathcal{V}\left(2 \omega_{0}\right) \oplus \bigoplus_{n \geq 1} \mathcal{V}\left(\omega_{n}\right)
$$

Remark. As in the proof below, the decomposition of $\mathcal{V}(\lambda) \otimes \mathcal{V}(\mu)$ can be obtained combinatorially by considering the highest weight vertices of $B(\lambda) \otimes B(\mu)$.

\section{The irreducible modules $V_{\infty}(\lambda)$}

In this section, we associate to each partition $\lambda$, an infinite dimensional irreducible $U_{q}\left(\mathfrak{g}_{\infty}\right)$-module $V_{\infty}(\lambda)$ which, contrary to the modules $\mathcal{V}(\lambda), \lambda \in P^{+}$of the previous section, is not a highest weight module.

3.1. Definition of $V_{\infty}(\lambda)$. Let $\mathcal{P}_{m}$ denote the set of partitions of length $m \in \mathbb{N}$ and set $\mathcal{P}=\bigcup_{m \in \mathbb{N}} \mathcal{P}_{m}$. In this paper it will be convenient to consider the partition $\lambda=\left(\lambda_{1}, \ldots, \lambda_{m}\right) \in \mathcal{P}_{m}$ as the increasing sequence of nonnegative integers $0 \leq \lambda_{1} \leq$ $\lambda_{2} \leq \cdots \leq \lambda_{m}$. For any integer $n \geq m$, one associates to $\lambda$ the dominant weight

$$
\phi_{n}(\lambda)=\sum_{i=1}^{m} \lambda_{i} \varepsilon_{i+n-m} \in P_{n}^{+} .
$$

Then we write $V_{n}(\lambda)$ for the finite dimensional $U_{q}\left(\mathfrak{g}_{n}\right)$-module of highest weight $\phi_{n}(\lambda)$. Consider two integers $r, s$ such that $m \leq r \leq s$. The restriction of $V_{s}(\lambda)$ from $U_{q}\left(\mathfrak{g}_{s}\right)$ to $U_{q}\left(\mathfrak{g}_{r}\right)$ contains a unique irreducible component isomorphic to $V_{r}(\lambda)$. Thus, there exists a unique embedding of $U_{q}\left(\mathfrak{g}_{r}\right)$-modules $f_{r, s}: V_{r}(\lambda) \rightarrow V_{s}(\lambda)$. Then we define $V_{\infty}(\lambda)$ as the direct limit of the direct system $\left(V_{r}(\lambda), f_{r, s}\right)$,

$$
V_{\infty}(\lambda)=\lim _{\rightarrow} V_{n}(\lambda)
$$

We can suppose without loss of generality that the $U_{q}\left(\mathfrak{g}_{n}\right)$-modules $V_{n}(\lambda)$ are chosen to verify $V_{n}(\lambda) \subset V_{n+1}(\lambda)$ for $n \geq m$. Under this hypothesis, the embeddings $f_{r, s}$ define above are trivial and we have $V_{\infty}(\lambda)=\bigcup_{n \geq m} V_{n}(\lambda)$.

Proposition 3.1.1. $V_{\infty}(\lambda)$ has the structure of an infinite dimensional irreducible $U_{q}\left(\mathfrak{g}_{\infty}\right)$-module.

Proof. Consider a Chevalley generator $g \in\left\{e_{i}, f_{i}, t_{i}, i \in I\right\}$ of $U_{q}\left(\mathfrak{g}_{\infty}\right)$ and a vector $v \in V_{\infty}(\lambda)$. Then there exists $N$ such that $v \in V_{N}(\lambda)$ and $g \in U_{q}\left(\mathfrak{g}_{N}\right)$. The action of $g$ on $v$ is then given by the structure of the $U_{q}\left(\mathfrak{g}_{N}\right)$-module on $V_{N}(\lambda)$. Since $V_{n}(\lambda) \subset V_{n+1}(\lambda)$ for any $n \geq m$, the action of $g$ on $v$ does not depend on the integer $N$ considered. One verifies easily that this defines the structure of a $U_{q}\left(\mathfrak{g}_{\infty}\right)$-module on $V_{\infty}(\lambda)$.

Suppose that $M$ is a sub- $U_{q}\left(\mathfrak{g}_{\infty}\right)$-module of $V_{\infty}(\lambda)$. For any positive integer $n \geq m=\ell(\lambda)$, write $M_{n}$ for the restriction of $M \cap V_{n}(\lambda)$ from $U_{q}\left(\mathfrak{g}_{\infty}\right)$ to $U_{q}\left(\mathfrak{g}_{n}\right)$. Then $M_{n}$ is a $U_{q}\left(\mathfrak{g}_{n}\right)$-submodule of $V_{n}(\lambda)$. Since $V_{n}(\lambda)$ is irreducible, we must have 
$M_{n}=\{0\}$ or $M_{n}=V_{n}(\lambda)$. If $M_{n}=\{0\}$ for each $n \geq m$, we obtain $M=\{0\}$ because $M=\bigcup_{n \geq 1} M_{n}$. Otherwise there exists an integer $n \geq m$ such that $M_{n}=$ $V_{n}(\lambda)$. Since $M_{n} \subset M_{n+1}$, this yields the equality $M_{p}=V_{p}(\lambda)$ for any integer $p \geq n$. So we obtain $M=\bigcup_{n \geq m} M_{n}=V_{\infty}(\lambda)$.

Remarks. (i) : Consider $\left(V_{r}(\lambda), f_{s, r}\right)$ and $\left(V_{r}^{\prime}(\lambda), f_{s, r}^{\prime}\right)$ two direct systems defined as above and their direct limits $V_{\infty}(\lambda)=\underline{\lim } V_{n}(\lambda), V_{\infty}^{\prime}(\lambda)=\underline{\lim } V_{n}^{\prime}(\lambda)$. For any $n \geq m$, let $\Psi_{n}$ be an isomorphism between $V_{n}(\lambda)$ and $V_{n}^{\prime}(\lambda)$. We must have $\Psi_{n+1}\left(V_{n}(\lambda)\right)=$ $V_{n}^{\prime}(\lambda)$ because $\Psi_{n+1}\left(V_{n}(\lambda)\right)$ is a $U_{q}\left(\mathfrak{g}_{n}\right)$-module isomorphic to $V_{n}(\lambda)$ contained in the restriction of $V_{n+1}(\lambda)$ to $U_{q}\left(\mathfrak{g}_{n}\right)$. Thus the map $\Psi: V_{\infty}(\lambda) \rightarrow V_{\infty}^{\prime}(\lambda)$ such that $\Psi(v)=\Psi_{n}(v)$ for any $v \in V_{n}(\lambda)$ is a well-defined isomorphism of $U_{q}\left(\mathfrak{g}_{\infty}\right)$-modules.

(ii) : Consider a weight vector $v \in V_{\infty}(\lambda)$ such that $v \in V_{m}(\lambda)$. Write $\operatorname{wt}_{m}(v)=$ $\left(\delta_{1}, \ldots, \delta_{m}\right) \in P_{m}$ for its weight. Then for any $n \geq m, v \in V_{n}(\lambda)$ is a vector of weight $\mathrm{wt}_{n}(v)=\left(\delta_{1}, \ldots, \delta_{n}, 0, \ldots, 0\right) \in P_{n}$. As a weight vector of $V_{\infty}(\lambda)$, the weight of $v$ can be written $\operatorname{wt}_{n}(v)=\left(\delta_{1}, \ldots, \delta_{n}, 0,0, \ldots\right) \in P$.

(iii) : For any partition $\lambda$, the weights of $V_{\infty}(\lambda)$ are elements of $P$ with a finite number of nonzero coordinates. They should not be confused with the highest weight modules $\mathcal{V}(\lambda)$ (where $\lambda \in P^{+}$was introduced in Section 2 whose weights have an infinite number of nonzero coordinates.

3.2. Littlewood-Richardson rule. Consider the infinite ordered alphabets

$$
\begin{aligned}
& \mathcal{X}_{A_{\infty}}=\{\cdots<\overline{2}<\overline{1}\}, \mathcal{X}_{B_{\infty}}=\{\cdots<\overline{2}<\overline{1}<0<1<2<\cdots\}, \\
& \mathcal{X}_{C_{\infty}}=\{\cdots<\overline{2}<\overline{1}<1<2<\cdots\}, \mathcal{X}_{D_{\infty}}=\left\{\cdots<\overline{2}<\frac{1}{1}<2<\cdots\right\} .
\end{aligned}
$$

Note that $\mathcal{X}_{D_{\infty}}$ is only partially ordered: 1 and $\overline{1}$ are not comparable. For any positive integer $n$, we denote by $\mathcal{X}_{n}$ the ordered alphabet obtained by considering only the letters of $\mathcal{X}_{\infty}$ which belong to $\{\bar{n}, \ldots, \overline{2}, \overline{1}, 0,1,2, \ldots, n\}$. Set

$$
\operatorname{wt}(\bar{i})=(\underbrace{0, \ldots, 0}_{i-1 \text { times }}, 1,0, \ldots) \in P, \operatorname{wt}(i)=(\underbrace{0, \ldots, 0}_{i-1 \text { times }},-1,0, \ldots) \in P \text { and } \operatorname{wt}(0)=0 \in P .
$$

The vector representation $V_{\infty}(1)$ of $U_{q}\left(\mathfrak{g}_{\infty}\right)$ is the vector space with basis $\left\{v_{x}\right.$, $\left.x \in \mathcal{X}_{\infty}\right\}$ where

$$
\begin{gathered}
q^{h}\left(v_{x}\right)=q^{\langle\operatorname{wt}(x), h\rangle} \text { for } h \in P^{*} \\
\left\{\begin{array}{l}
f_{i}\left(v_{\overline{i+1}}\right)=v_{\bar{i}}, f_{i}\left(v_{i}\right)=v_{i+1} \text { and } f_{i}\left(v_{x}\right)=0 \text { if } x \notin\{\overline{i+1}, i\} \\
e_{i}\left(v_{\bar{i}}\right)=v_{\overline{i+1}}, f_{i}\left(v_{i+1}\right)=v_{i} \text { and } e_{i}\left(v_{x}\right)=0 \text { if } x \notin\{\bar{i}, i+1\}
\end{array} \quad \text { if } i \neq 0\right.
\end{gathered}
$$

and the action of $e_{0}, f_{0}$ depends on the type considered:

$$
\begin{aligned}
& \left\{\begin{array}{l}
e_{0}\left(v_{1}\right)=v_{0}, e_{0}\left(v_{0}\right)=\left(q+q^{-1}\right) v_{\overline{1}} \text { and } e_{0}\left(v_{x}\right)=0 \text { if } x \notin\{0,1\} \\
f_{0}\left(v_{\overline{1}}\right)=v_{0}, f_{0}\left(v_{0}\right)=\left(q+q^{-1}\right) v_{1} \text { and } f_{0}\left(v_{x}\right)=0 \text { if } x \notin\{\overline{1}, 0\}
\end{array} \text { for type } B_{\infty},\right. \\
& \left\{\begin{array}{l}
e_{0}\left(v_{1}\right)=v_{\overline{1}} \text { and } e_{0}\left(v_{x}\right)=0 \text { if } x \neq 1 \\
f_{0}\left(v_{\overline{1}}\right)=v_{1} \text { and } f_{0}\left(v_{x}\right)=0 \text { if } x \neq \overline{1}
\end{array} \text { for type } C_{\infty},\right. \\
& \left\{\begin{array}{l}
f_{0}\left(v_{\overline{2}}\right)=v_{1}, f_{0}\left(v_{\bar{i}}\right)=v_{2} \text { and } f_{0}\left(v_{x}\right)=0 \text { if } x \notin\{\overline{2}, \overline{1}\} \\
e_{i}\left(v_{2}\right)=v_{\overline{1}}, f_{i}\left(v_{1}\right)=v_{\overline{2}} \text { and } e_{0}\left(v_{x}\right)=0 \text { if } x \notin\{1,2\}
\end{array} \text { for type } D_{\infty} .\right.
\end{aligned}
$$

For any positive integer $n$, the $U_{q}\left(\mathfrak{g}_{n}\right)$-module $V_{n}(1)$ is identified with the vector space with basis $\left\{v_{x}, x \in \mathcal{X}_{n}\right\}$ with the action of the Chevalley generators $e_{i}, f_{i}, t_{i}$, $i \in I_{n}$. 
For any nonnegative integer $\ell$, there exists a finite number of irreducible modules $V_{n}^{(1)}, \ldots, V_{n}^{(k)}$ such that

$$
V_{n}(1)^{\otimes \ell}=\bigoplus_{k=1}^{r} V_{n}^{(k)} .
$$

Set $E_{n, \ell}=\left\{k \in\{1, \ldots, r\} \mid V_{n}^{(k)} \simeq V_{n}(\lambda)\right.$ with $\left.|\lambda|=\ell\right\}$ and $F_{n, \ell}=\{1, \ldots, r\}-E_{n, \ell}$. Then consider the submodules $M_{n, \ell}$ and $N_{n, \ell}$ defined by

$$
M_{n, \ell}=\bigoplus_{k \in E_{n, \ell}} V_{n}^{(k)} \text { and } N_{n, \ell}=\bigoplus_{k \in F_{n, \ell}} V_{n}^{(k)} \text {. }
$$

These modules do not depend on the decomposition (13) and we have $V_{n}(1)^{\otimes \ell}=$ $M_{n, \ell} \oplus N_{n, \ell}$. The module $M_{n, \ell}$ (resp. $\left.N_{n, \ell}\right)$ contains all the highest weight vectors of highest weight $\lambda$ verifying $|\lambda|=\ell$ (resp. $|\lambda|<\ell$ ). Note that $N_{n, \ell}=\{0\}$ only for type $A_{n-1}$.

The crystal basis of $V_{n}(1)$ is the pair $\left(L_{n}(1), B_{n, 1}\right)$ where

$$
L_{n}(1)=\bigoplus_{x \in \mathcal{X}_{n}} \mathbf{A}(q) v_{x}
$$

and for types $A_{n-1}, B_{n}, C_{n}, D_{n}$, the crystals $B_{n, 1}$ are respectively

$$
\begin{aligned}
& \bar{n} \stackrel{n-1}{\rightarrow} \overline{n-1} \stackrel{n-2}{\rightarrow} \cdots \rightarrow \overline{2} \stackrel{1}{\rightarrow} \overline{1} \\
& \bar{n} \stackrel{n-1}{\rightarrow} \overline{n-1} \stackrel{n-2}{\rightarrow} \cdots \rightarrow \overline{2} \stackrel{1}{\rightarrow} \overline{1} \stackrel{0}{\rightarrow} 0 \stackrel{0}{\rightarrow} 1 \stackrel{1}{\rightarrow} 2 \cdots \stackrel{n-2}{\rightarrow} n-1 \stackrel{n-1}{\rightarrow} n \\
& \bar{n} \stackrel{n-1}{\rightarrow} \overline{n-1} \stackrel{n-2}{\rightarrow} \cdots . \overline{2} \stackrel{1}{\rightarrow} \overline{1} \stackrel{0}{\rightarrow} 1 \stackrel{1}{\rightarrow} 2 \cdots \stackrel{n-2}{\rightarrow} n-1 \stackrel{n-1}{\rightarrow} n \\
& 1 \\
& \bar{n} \stackrel{n-1}{\rightarrow} \frac{n-1}{n-2} \rightarrow \stackrel{3}{\rightarrow} \overline{3} \stackrel{2}{\rightarrow} \overline{2} \nearrow_{1}^{\searrow} \nearrow_{\overline{1}}^{0} \searrow^{\searrow} 2 \stackrel{2}{\rightarrow} 3 \stackrel{3}{\rightarrow} \cdots \stackrel{n-2}{\rightarrow} n-1 \stackrel{n-1}{\rightarrow} n .
\end{aligned}
$$

The crystal basis of $V_{n}(1)^{\otimes \ell}$ can be realized as the pair $\left(L_{n, \ell}, B_{n, \ell}\right)$ with $L_{n, \ell}=$ $\bigoplus \mathbf{A}(q) v_{x_{1}} \otimes \cdots \otimes v_{x_{\ell}}$ and $B_{n, \ell}=B_{n, 1}^{\otimes \ell}$. The weight of the vertex $b=x_{1} \otimes \cdots \otimes x_{\ell}$ is the $n$-tuple $\operatorname{wt}_{n}(b)=\left(d_{1}, \ldots, d_{n}\right) \in P_{n}$ where for any $i \in I_{n}, d_{i}$ is the number of letters $x_{k}=\bar{i}$ in $b$ minus its number of letters $x_{k}=i$. In particular $\left|\mathrm{wt}_{n}(b)\right|$ is the number of barred letters in $b$ minus its number of unbarred letters. The pair

$$
L_{n, \ell} \cap N_{n, \ell}, B_{n, l}^{N}=\left\{b+q L_{n, \ell} \cap N_{n, \ell} \mid b+q L_{n, \ell} \in B_{n, \ell}, b \in L_{n, \ell} \cap N_{n, \ell}\right\}
$$

is a crystal basis of $N_{n, \ell}$. This implies that $B_{n, \ell}^{N}$ is the subcrystal of $B_{n, \ell}$ containing all the connected components isomorphic to $B_{n}(\lambda)$ with $|\lambda|<\ell$.

Given $m \leq n$ two integers, denote by $\rho_{m, n}: V_{n}(1)^{\otimes \ell} \rightarrow V_{m}(1)^{\otimes \ell}$ the canonical projection. Then the image of the crystal basis $\left(L_{n, \ell}, B_{n, \ell}\right)$ by $\rho_{m, n}$ is the crystal basis $\left(L_{m, \ell}, B_{m, \ell}\right)$.

We now establish three lemmas which are essential to obtain the independence of the root system in Theorem 3.2.4 and Corollary 3.2.5.

Lemma 3.2.1. Consider $m$ a nonnegative integer. Then for $n \geq m$ sufficiently large, $B_{m, \ell} \cap B_{n, \ell}^{N}=\emptyset$. 
Proof. For any $b \in B_{m, \ell}$, denote by $B_{n, \ell}(b)$ the connected component of $B_{n, \ell}$ containing $b$. Let $b_{h}$ be the highest weight vertex of $B_{n, \ell}(b)$. We are going to prove by induction on $\ell$ that for $n$ sufficiently large, there exists a partition $\lambda$ such that $\operatorname{wt}\left(b_{h}\right)=\phi_{n}(\lambda)$ with $|\lambda|=\ell$.

For $\ell=1$, this is immediate because $b_{h}=\bar{n}$ has weight $\phi_{n}(1)$. So, consider $b \in B_{m, \ell}, \ell \geq 2$ and write $b=b^{b} \otimes x_{\ell}, b_{h}=b_{h}^{b} \otimes x_{\ell, h}$ where $b^{b}, b_{h}^{b} \in B_{n, \ell-1}$ and $x_{\ell}, x_{\ell, h} \in B_{n, 1}$. Then it follows from (8) and Lemma 2.2.1 that $b_{h}^{b}$ is the highest weight vertex of $B_{n, \ell-1}\left(b^{b}\right)$. Choose $n \geq \ell$ sufficiently large so that there exists a partition $\gamma$ verifying $\operatorname{wt}\left(b^{b}\right)=\phi_{n}(\gamma)$ with $|\gamma|=\ell-1$. The letters of $b_{h}^{b}$ belong to $\{\bar{n}, \ldots, \overline{n-\ell+2}\}$ for $|\gamma|=\ell-1$ and $\phi_{n}(\gamma) \in P_{n-1}^{+}$. Moreover for any $i \in I_{n}$, we must have $\varepsilon_{i}\left(x_{\ell, h}\right) \leq \varphi_{i}\left(b_{h}^{b}\right)$. Since $b \in B_{m, \ell}$ and $x_{\ell, h} \leq x_{\ell}$, we have $x_{\ell, h} \leq m$. Suppose that $x_{\ell, h}=k+1$ with $k \in\{0, \ldots, m-1\}$. Then $\varepsilon_{k}\left(x_{\ell, h}\right)=1$; thus $\varphi_{k}\left(b_{h}^{b}\right) \geq 1$. This implies that $b_{h}^{b}$ contains at least a letter $\overline{k+1}$. Hence we obtain $n-\ell+1 \leq k \leq m-1$. This means that $n$ can be chosen sufficiently large so that $b_{h}^{b}$ has weight $\phi_{n}(\gamma)$ with $\gamma \in \mathcal{P},|\gamma|=\ell-1$ and $x_{\ell, h}$ is barred. For such an integer $n, b_{h}$ contains only barred letters. Thus there exists a partition $\lambda$ such that $\phi_{n}(\lambda)=\operatorname{wt}\left(b_{h}\right)$ and $|\lambda|=\ell$.

Lemma 3.2.2. For any positive integer $m$, there exists an integer $n \geq m$ such that $V_{m}(1)^{\otimes \ell}$ is a submodule of the restriction of $M_{n, \ell}$ to $U_{q}\left(\mathfrak{g}_{m}\right)$.

Proof. From the decomposition $V_{n}(1)^{\otimes \ell}=M_{n, \ell} \oplus N_{n, \ell}$, we obtain $V_{m}(1)^{\otimes \ell}=M_{n, \ell} \cap$ $V_{m}(1)^{\otimes \ell} \oplus N_{n, \ell} \cap V_{m}(1)^{\otimes \ell}$ for any $m \leq n$. Since $L_{m, \ell}=L_{n, \ell} \cap V_{m}(1)^{\otimes \ell}$, we derive from (14) that the pair

$L_{m, \ell} \cap N_{n, \ell}$ and $B_{m, n, l}^{N}=\left\{b+q L_{m, \ell} \cap N_{n, \ell} \mid b+q L_{n, \ell} \cap N_{n, \ell} \in B_{n, \ell}^{N}, b \in L_{m, \ell} \cap N_{n, \ell}\right\}$

is a crystal basis of the $U_{q}\left(\mathfrak{g}_{m}\right)$-module $N_{n, \ell} \cap V_{m}(1)^{\otimes \ell}$. In particular, the vertices of $B_{m, n, l}^{N}$ are the vertices of $B_{m, \ell}$ which belong to $B_{n, \ell}^{N}$. By the above lemma, we obtain that $B_{m, n, l}^{N}=\emptyset$ for $n$ sufficiently large. For such an integer $n$, we must have $N_{n, \ell} \cap V_{m}(1)^{\otimes \ell}=\{0\}$ because the dimension of $N_{n, \ell} \cap V_{m}(1)^{\otimes \ell}$ is equal to the number of vertices of $B_{m, n, l}^{N}$. Finally, this gives $V_{m}(1)^{\otimes \ell}=M_{n, \ell} \cap V_{m}(1)^{\otimes \ell}$; thus $V_{m}(1)^{\otimes \ell}$ is a submodule of the restriction of $M_{n, \ell}$ to $U_{q}\left(\mathfrak{g}_{m}\right)$.

For any $x \in \mathcal{X}$, set

$$
\theta(x)=\left\{\begin{array}{l}
i+1 \text { if } x=i \text { is unbarred } \\
\overline{i+1} \text { if } x=\bar{i} \text { is barred. }
\end{array}\right.
$$

We also denote by $\theta$ the linear map defined on $V_{\infty}(1)^{\otimes \ell}$ by setting $\theta\left(v_{x_{1}} \otimes \cdots \otimes v_{x_{\ell}}\right)=$ $v_{\theta\left(x_{1}\right)} \otimes \cdots \otimes v_{\theta\left(x_{\ell}\right)}$.

Lemma 3.2.3. Let $v$ be vector in $V_{\infty}(1)^{\otimes \ell}$ of weight $\delta=\left(\delta_{1}, \ldots, \delta_{n}, 0, \ldots\right) \in P$, where $\delta_{n} \neq 0$. Suppose that $|\delta|=\ell$. Then, there exists $K(q)$ in $\mathbb{Q}(q)$ such that

$$
f_{n}^{\delta_{n}} \cdots f_{1}^{\delta_{1}}(\theta(v))=K(q) v .
$$

Proof. Observe first that $\theta(v) \in V_{\infty}(1)^{\otimes \ell}$ is a vector of weight $\left(0, \delta_{1}, \ldots, \delta_{n}, 0, \ldots\right) \in$ $P$. Since $\delta_{1}+\cdots+\delta_{n}=\ell$, each tensor $v_{x_{1}} \otimes \cdots \otimes v_{x_{\ell}}$ appearing in its decomposition

$$
\theta(v)=\sum a_{x_{1} \cdots x_{\ell}}(q) v_{x_{1}} \otimes \cdots \otimes v_{x_{\ell}}
$$


on the tensor basis of $V_{\infty}(1)^{\otimes \ell}$ must verify $x_{k} \in\{\overline{2}, \ldots, \overline{n+1}\}$. Then by (4) there exists $K_{1}(q)$ in $\mathbb{Q}(q)$ such that

$$
f_{1}^{\delta_{1}}(\theta(v))=K_{1}(q) v^{(1)},
$$

where $v^{(1)}$ is obtained by replacing each factor $v_{\overline{2}}$ appearing in (15) by a factor $v_{\overline{1}}$. Indeed the factors $v_{x_{k}}$ with $x_{k} \neq \overline{2}$ do not interfere with the computation of $f_{1}^{\delta_{1}}(v)$. Similarly the tensors appearing in the decomposition of $v^{(1)}$ on the tensor basis have factors in $\{\overline{1}, \overline{3}, \ldots, \overline{n+1}\}$. Hence there also exists $K_{2}(q)$ in $\mathbb{Q}(q)$ such that $f_{2}^{\delta_{2}}\left(v^{(1)}\right)=K_{2}(q) v^{(2)}$ where $v^{(2)}$ is obtained by replacing each factor $v_{\overline{3}}$ appearing in $v^{(1)}$ by a factor $v_{\overline{2}}$ (the other factors do not interfere with the computation of $\left.f_{2}^{\delta_{2}}\left(v^{(1)}\right)\right)$. The lemma follows by an easy induction.

Theorem 3.2.4. For any integer $\ell, V_{\infty}(1)^{\otimes \ell}$ decomposes into irreducible components following

$$
V_{\infty}(1)^{\otimes \ell} \simeq \bigoplus_{\nu \in \mathcal{P},|\nu|=\ell} V_{\infty}(\nu)^{\oplus n_{\nu}},
$$

where $n_{\nu}$ is the number of standard tableaux of shape $\nu$.

Proof. The dimension of the space generated by the highest weight vectors in $V_{\ell}(1)^{\otimes \ell}$ of weight $\phi_{\ell}(\nu) \in P_{\ell}$ such that $|\nu|=\ell$ is equal to the number of standard tableaux of shape $\nu$. Indeed, there is a bijection between these vectors and the Yamanouchi words of length $\ell$ [12. Fix a basis $\left\{v_{t}^{\ell} \mid t \in \mathrm{ST}(\ell)\right\}$ (labelled by the standard tableaux with $\ell$ boxes) of highest weight vectors in $V_{\ell}(1)^{\otimes \ell}$ having a weight of the form $\phi_{\ell}(\nu)$ with $|\nu|=\ell$. Consider an integer $n \geq \ell$ and a standard tableau $t \in \mathrm{ST}(\ell)$ of shape $\nu$. Since $|\nu|=\ell$, the decomposition of $v_{t}^{\ell}$ on the tensor basis of $V_{\ell}(1)^{\otimes \ell}$ causes only those tensors $v_{x_{1}} \otimes \cdots \otimes v_{x_{\ell}}$ such that $x_{k}$ is barred for any $k \in\{1, \ldots, \ell\}$, to appear.

By (5), $\theta^{(n-\ell)}\left(v_{t}^{\ell}\right)=v_{t}^{n}$ is a highest weight vector of $V_{n}(1)^{\otimes \ell}$ of weight $\phi_{n}(\nu) \in P_{n}$ such that $|\nu|=\ell$. This permits us to define a $U_{q}\left(\mathfrak{g}_{n}\right)$-module $V_{n}(t)=U_{q}\left(\mathfrak{g}_{n}\right) \cdot v_{t}^{n}$ isomorphic to $V_{n}(\nu)$. By Lemma 3.2.3 there exists $K(q) \in \mathbb{Q}(q)$ such that

$$
f_{n}^{\nu_{p}} \cdots f_{n-p+1}^{\nu_{1}}\left(v_{t}^{n+1}\right)=K(q) v_{t}^{n} .
$$

This gives the inclusion $V_{n}(t) \subset V_{n+1}(t)$. Hence $V_{\infty}(t)=\bigcup_{n \geq \ell} V_{n}(t)$ is isomorphic to $V_{\infty}(\nu)$.

Since $\left\{v_{t}^{\ell} \mid t \in \mathrm{ST}(\ell)\right\}$ is a basis of highest weight vectors in $V_{\ell}(1)^{\otimes \ell}$ of weight $\phi_{\ell}(\nu)$ with $|\nu|=\ell$, for any $n \geq \ell,\left\{v_{t}^{n} \mid t \in \mathrm{ST}(\ell)\right\}$ is a basis of highest weight vectors in $V_{n}(1)^{\otimes \ell}$ of weight $\phi_{n}(\nu)$ with $|\nu|=\ell$. Thus $M_{n, \ell}=\bigoplus_{t \in \mathrm{ST}(\ell)} V_{n}(t)$. Set $M_{\ell}=\bigoplus_{t \in \mathrm{ST}(\ell)} V_{\infty}(t)$. Then $M_{\ell}=\bigcup_{n \geq \ell} M_{n, \ell}$. For any integer $m \geq \ell$, we derive by Lemma 3.2 .2 that there exists an integer $n \geq m$ such that $V_{m}(1)^{\otimes \ell} \subset M_{n, \ell} \subset M_{\ell}$. Since $V_{\infty}(1)^{\otimes \ell}=\bigcup_{m \geq \ell} V_{m}(1)^{\otimes \ell}$, this gives $V_{\infty}(1)^{\otimes \ell} \subset M_{\ell} \subset V_{\infty}(1)^{\otimes \ell}$. Hence $V_{\infty}(1)^{\otimes \ell}=M_{\ell}$ and the theorem is proved for $M_{\ell}$ is isomorphic by definition to $\bigoplus_{\nu \in \mathcal{P},|\nu|=\ell} V_{\infty}(\nu)^{\oplus n_{\nu}}$.

Consider $\nu \in \mathcal{P}_{m}$. For any integer $\ell \geq m$, let $v_{\nu}^{\ell} \in V_{\ell}(1)^{\otimes \ell}$ be a highest weight vector of weight $\phi_{\ell}(\nu)$ such that $|\nu|=\ell$. Similarly to the previous proof, for any integer $n \geq \ell$ the vector $v_{\nu}^{n}=\theta^{(n-\ell)}\left(v_{\nu}^{\ell}\right)$ is of highest weight $\phi_{n}(\nu)$ in $V_{n}(1)^{\otimes \ell}$. By setting $V_{n}\left(v_{\nu}^{\ell}\right)=U_{q}\left(\mathfrak{g}_{n}\right) \cdot v_{\nu}^{n}$ for any $\ell \geq n$, one defines the module $V_{\infty}\left(v_{\nu}^{\ell}\right)=$ $\bigcup_{n \geq \ell} V_{n}\left(v_{\nu}^{\ell}\right)$ isomorphic to $V_{\infty}(\nu)$. Set $\rho_{n}\left(V_{\infty}\left(v_{\nu}^{\ell}\right)\right)=V_{\infty}\left(v_{\nu}^{\ell}\right) \cap V_{n}(1)^{\otimes \ell}$. Then 
$\rho_{n}\left(V_{\infty}\left(v_{\nu}^{\ell}\right)\right)$ has the structure of a $U_{q}\left(\mathfrak{g}_{n}\right)$-module. Since $V_{\infty}\left(v_{\nu}^{\ell}\right)=\bigcup_{n \geq \ell} V_{n}\left(v_{\nu}^{\ell}\right)$, there exists an integer $p \geq n$ such that $\rho_{n}\left(V_{\infty}\left(v_{\nu}^{\ell}\right)\right)$ is a submodule of the restriction of $V_{p}\left(v_{\nu}^{\ell}\right)$ from $U_{q}\left(\mathfrak{g}_{p}\right)$ to $U_{q}\left(\mathfrak{g}_{n}\right)$. Thus the decomposition of $\rho_{n}\left(V_{\infty}\left(v_{\nu}^{\ell}\right)\right)$ verifies

$$
\rho_{n}\left(V_{\infty}\left(v_{\nu}^{\ell}\right)\right) \simeq V_{n}(\nu) \oplus \bigoplus_{\lambda \in \mathcal{P}|, \lambda|<\ell} V_{n}(\lambda)^{\oplus m_{\nu, \lambda}\left(v_{\nu}^{\ell}\right)},
$$

where the multiplicities $m_{\nu, \lambda}\left(v_{\nu}^{\ell}\right)$ depend on $v_{\nu}^{\ell}$ in general.

Corollary 3.2.5. Consider $\lambda$ and $\mu$ two partitions respectively of lengths $m$ and $n$. Then we have the following decomposition:

$$
V_{\infty}(\lambda) \otimes V_{\infty}(\mu) \simeq \bigoplus_{\nu \in \mathcal{P}} V_{\infty}(\nu)^{\oplus c_{\lambda, \mu}^{\nu}}
$$

where $c_{\lambda, \mu}^{\nu}$ is the Littlewood-Richardson coefficient corresponding to the partitions $\lambda, \mu$ and $\nu$.

Proof. Set $\ell=|\lambda|+|\nu|$. We realize the modules $V_{\infty}(\lambda)$ and $V_{\infty}(\mu)$ as irreducible components respectively of $V_{\infty}(1)^{\otimes|\lambda|}$ and $V_{\infty}(1)^{\otimes|\mu|}$. Then, by the previous theorem, the tensor product $V_{\infty}(\lambda) \otimes V_{\infty}(\mu) \subset V_{\infty}(1)^{\otimes \ell}$ decomposes in the form

$$
V_{\infty}(\lambda) \otimes V_{\infty}(\mu) \simeq \bigoplus_{\nu \in \mathcal{P},|\nu|=|\lambda|+|\mu|} V_{\infty}(\nu)^{\oplus d_{\lambda, \mu}^{\nu}}
$$

For any $\nu \in \mathcal{P}$ such that $|\nu|=\ell$ and any integer $n \geq \ell$, we derive from (17) that $\rho_{n}\left(V_{\infty}(\lambda) \otimes V_{\infty}(\mu)\right)=\rho_{n}\left(V_{\infty}(\lambda)\right) \otimes \rho_{n}\left(V_{\infty}(\mu)\right)$ contains exactly $c_{\lambda, \mu}^{\nu}$ irreducible components isomorphic to $V_{n}(\nu)$. By comparing with the right hand side of (18) and using (17) once again, we obtain $d_{\lambda, \mu}^{\nu}=c_{\lambda, \mu}^{\nu}$.

In the sequel we denote by $\mathcal{M}$ the category of modules $M$ which can be realized as submodules of a tensor power $V_{\infty}(1)^{\otimes \ell}$. By the corollary, $\mathcal{M}$ is closed under taking direct sums or tensor products of finitely many $U_{q}\left(\mathfrak{g}_{\infty}\right)$-modules. Moreover any module in $\mathcal{M}$ is isomorphic to a direct sum of irreducible $U_{q}\left(\mathfrak{g}_{\infty}\right)$-modules $V_{\infty}(\lambda)$ with $\lambda \in \mathcal{P}$.

3.3. Explicit realization of the modules $V_{\infty}(\lambda)$. By Theorem 3.2.4, we have the decomposition

$$
V_{\infty}(1)^{\otimes 2} \cong V_{\infty}(2) \oplus V_{\infty}(1,1) .
$$

For any positive integer $p$, set

$$
N_{p}=\sum_{i=0}^{p-2} V_{\infty}(1)^{\otimes i} \otimes V_{\infty}(2) \otimes V_{\infty}(1)^{\otimes(p-2-i)}
$$

(where the sum is not direct).

Proposition 3.3.1. With the above notation,

$$
V_{\infty}\left(1^{p}\right) \simeq V_{\infty}(1)^{\otimes p} / N_{p} .
$$

Proof. By Theorem 3.2.4 and Corollary 3.2.5 we have

$$
V_{\infty}(1)^{\otimes p} \simeq \bigoplus_{\nu \in \mathcal{P}_{p}} V_{\infty}(\nu)^{\oplus n_{\nu}} \text { and } N_{p} \simeq \bigoplus_{\nu \in \mathcal{P}_{p}^{*}} V_{\infty}(\nu)^{\oplus n_{\nu}},
$$

where $\mathcal{P}_{p}^{*}$ is the set of partitions of length $p$ with at least a part $\geq 2$. Since $\mathcal{P}_{p}=$ $\mathcal{P}_{p}^{*} \cup\left\{\left(1^{p}\right)\right\}$, this implies the proposition. 
The representation $V_{\infty}\left(1^{p}\right)$ can thus be regarded as a $q$-analogue of the $p$-th wedge product of $V_{\infty}(1)$. Let $\Psi_{p}$ be the canonical projection $V_{\infty}(1)^{\otimes p} \rightarrow V_{\infty}\left(1^{p}\right)$. Denote by $v_{x_{1}} \wedge \cdots \wedge v_{x_{p}}$ the image of the vector $v_{x_{1}} \otimes \cdots \otimes v_{x_{p}}$ by $\Psi_{p}$. The following lemma is proved as Proposition 3.1.1 in [17]:

Lemma 3.3.2. For type $B_{\infty}$, we have in $V_{\infty}\left(1^{2}\right)$ the relations

(1) $v_{x} \wedge v_{x}=0$ for $x \neq 0$,

(2) $v_{y} \wedge v_{x}=-q^{2} v_{x} \wedge v_{y}$ for $x \neq \bar{y}$ and $x<y$,

(3) $v_{i} \wedge v_{\bar{i}}=-q^{4} v_{\bar{i}} \wedge v_{i}+\left(1-q^{4}\right) \sum_{k=1}^{i-1}(-1)^{i-k} q^{2(i-k)} v_{\bar{k}} \wedge v_{k}+(-1)^{i} q^{2 i-1} v_{0} \wedge v_{0}$ for $i=1, \ldots, n$.

For type $C_{\infty}$, we have in $V_{\infty}\left(1^{2}\right)$ the relations

(1) $v_{x} \wedge v_{x}=0$ for $x \neq 0$,

(2) $v_{y} \wedge v_{x}=-q v_{x} \wedge v_{y}$ for $x \neq \bar{y}$ and $x<y$,

(3) $v_{i} \wedge v_{\bar{i}}=-q^{2} v_{\bar{i}} \wedge v_{i}+\left(1-q^{2}\right) \sum_{k=1}^{i-1}(-1)^{i-k} q^{i-k} v_{\bar{k}} \wedge v_{k}$ for $i=1, \ldots, n$.

For type $D_{\infty}$, we have in $V_{\infty}\left(1^{2}\right)$ the relations

(1) $v_{x} \wedge v_{x}=0$ for $x \neq 0$,

(2) $v_{y} \wedge v_{x}=-q v_{x} \wedge v_{y}$ for $x \neq \bar{y}$ and $x<y$,

(3) $v_{i} \wedge v_{\bar{i}}=-q^{2} v_{\bar{i}} \wedge v_{i}+\left(1-q^{2}\right) \sum_{k=2}^{i-1}(-1)^{i-k} q^{i-k} v_{\bar{k}} \wedge v_{k}+(-q)^{i-1}\left(v_{1} \wedge v_{\overline{1}}+v_{\overline{1}} \wedge v_{1}\right)$ for $i=2, \ldots, n$.

The columns of types $A_{\infty}, B_{\infty}, C_{\infty}$ and $D_{\infty}$ are Young diagrams $C$ of shape column respectively of the form

$$
C=C_{-}, C=\begin{array}{|l|}
\hline C_{-} \\
\hline C_{0} \\
\hline C_{+}
\end{array}, C=\begin{array}{|l|}
\hline C_{-} \\
\hline C_{+} \\
\hline
\end{array} \text { and } C=\begin{array}{c|}
\hline D_{-} \\
\hline D \\
\hline D_{+} \\
\hline
\end{array}
$$

where $C_{-}, C_{+}, C_{0}, D_{-}, D_{+}$and $D$ are column shaped Young diagrams such that

$$
\left\{\begin{array}{l}
C_{-} \text {is filled by strictly increasing barred letters from top to bottom, } \\
C_{+} \text {is filled by strictly increasing unbarred letters from top to bottom, } \\
C_{0} \text { is filled by letters } 0, \\
D_{-} \text {is filled by strictly increasing letters } \leq \overline{2} \text { from top to bottom, } \\
D_{+} \text {is filled by strictly increasing letters } \geq 2 \text { from top to bottom, } \\
D \text { is filled by letters } \overline{1} \text { or } 1 \text { with different letters in two adjacent boxes. }
\end{array}\right.
$$

We denote by $\mathbf{C}(p)$ the set of columns of height $p$. The reading of the column $C$ is the word w $(C)$ obtained by reading the letters of $C$ from top to bottom. For any column $C$ with w $(C)=c_{1} \cdots c_{p}$ where the $c_{i}$ 's are letters, we set $v_{C}=v_{c_{1}} \wedge \cdots \wedge v_{c_{p}}$. Then each vector $\Psi_{p}\left(v_{x_{1}} \otimes \cdots \otimes v_{x_{p}}\right)=v_{x_{1}} \wedge \cdots \wedge v_{x_{p}}$ can be decomposed into a linear combination of vectors $v_{C}$ by applying from left to right a sequence of relations given in the above lemma.

Proposition 3.3.3. The vectors of $\left\{v_{C}, C \in \mathbf{C}(p)\right\}$ form a basis of $V_{\infty}\left(1^{p}\right)$.

Proof. By Lemma 3.3.2, each vector of $V_{\infty}\left(1^{p}\right)$ can be decomposed into a linear combination of vectors $v_{C}$. Given a finite family $\left\{v_{i}\right\}_{i \in I}$ of vectors $v_{C}$, there exists an integer $n$ such that all the vectors in $\left\{v_{i}\right\}_{i \in I}$ belong to $V_{n}\left(1^{p}\right)$. By Lemma 
3.1 .2 of [17], the vectors of $\left\{v_{i}\right\}_{i \in I}$ are linearly independent in $V_{n}\left(1^{p}\right)$, thus also in $V_{\infty}\left(1^{p}\right)$.

Consider $\lambda \in \mathcal{P}_{m}$ for any $p \in\{1, \ldots, m\}$ and denote by $\widehat{\lambda}_{p}$ the number of columns of height $p$ in the Young diagram of $\lambda$. Set

$$
W_{\infty}(\lambda)=V_{\infty}(1)^{\otimes \widehat{\lambda}_{1}} \otimes \cdots \otimes V_{\infty}\left(1^{m}\right)^{\otimes \widehat{\lambda}_{m}}
$$

The natural basis of $W_{\infty}(\lambda)$ consists of the tensor products $v_{C_{r}} \otimes \cdots \otimes v_{C_{1}}$ of basis vectors $v_{C}$ of the previous section appearing in (21). For any positive integer $n \geq m$ set $u_{p}^{(n)}=v_{n-p+1} \wedge \cdots \wedge v_{\bar{n}}$. The vector $u_{p}^{(n)}$ is of highest weight $\phi_{n}\left(1^{p}\right)$ in the restriction of $V_{\infty}\left(1^{p}\right)$ to $U_{q}\left(\mathfrak{g}_{n}\right)$. In the restriction of $W_{\infty}(\lambda)$ to $U_{q}\left(\mathfrak{g}_{n}\right)$, the vector $u_{\lambda}^{(n)}=\left(u_{1}^{(n)}\right)^{\otimes \widehat{\lambda}_{1}} \otimes \cdots \otimes\left(u_{m}^{(n)}\right)^{\otimes \widehat{\lambda}_{m}}$ is the highest weight vector of an irreducible component $V\left(u_{\lambda}^{(n)}\right)$ isomorphic to $V_{n}(\lambda)$. From Lemma 3.2.3, we derive $V\left(u_{\lambda}^{(n)}\right) \subset V\left(u_{\lambda}^{(n+1)}\right)$. By Remark (i) after Proposition 3.1.1, we obtain:

Proposition 3.3.4. The $U_{q}\left(\mathfrak{g}_{\infty}\right)$-modules $\bigcup_{n \geq m} V\left(u_{\lambda}^{(n)}\right)$ and $V_{\infty}(\lambda)$ are isomorphic.

\section{Crystal basis And CANONical Basis for the modules in $\mathcal{M}$}

4.1. Crystal basis. Consider $M$ a module in the category $\mathcal{M}$. Since $M$ is decomposable, there exists a finite number of irreducible $U_{q}\left(\mathfrak{g}_{\infty}\right)$-modules $V_{\infty}^{(1)}, \ldots, V_{\infty}^{(r)}$ in $\mathcal{M}$ such that

$$
M=\bigoplus_{k=1}^{r} V_{\infty}^{(k)} .
$$

Each irreducible module $V_{\infty}^{(k)}$ in $\mathcal{M}$ being isomorphic to a module $V_{\infty}(\lambda)$ with $\lambda \in \mathcal{P}$, its restriction to $U_{q}\left(\mathfrak{g}_{n}\right)$ contains a unique irreducible component $V_{n}^{(k)}$ isomorphic to $V_{n}(\lambda)$. Then we set $M_{n}=\bigoplus_{1 \leq k \leq r} V_{n}^{(k)}$. We have $M_{n} \subset M_{n+1}$ and $M=\bigcup_{n \geq 1} M_{n}$.

Remark. Consider $M$ and $N$ two $U_{q}\left(\mathfrak{g}_{\infty}\right)$-modules. For types $B, C$ and $D,(M \otimes N)_{n}$ is a submodule of $M_{n} \otimes N_{n}$ but we have $(M \otimes N)_{n} \neq M_{n} \otimes N_{n}$ in general. When $M=V_{\infty}(\lambda)$ and $N=V_{\infty}(\mu)$ are irreducible, $\left(V_{\infty}(\lambda) \otimes V_{\infty}(\nu)\right)_{n}$ is the submodule of $V_{n}(\lambda) \otimes V_{n}(\nu)$ obtained as the direct sum of its irreducible components of highest weight $\phi_{n}(\nu)$ where $|\nu|=|\lambda|+|\mu|$ (see Corollary 3.2.5) .

Recall that each irreducible module $V_{n}(\lambda)$ has a crystal basis $\left(L_{n}(\lambda), B_{n}(\lambda)\right)$ unique up to the choice of a highest weight $u_{\lambda}$ vector in $V_{n}(\lambda)$. Indeed, $L_{n}(\lambda)$ is the $\mathbf{A}(q)$-submodule of $V_{n}(\lambda)$ spanned by the vectors of the form $\tilde{f}_{i_{1}} \cdots \widetilde{f}_{i_{r}} u_{\lambda}$ and

$$
B_{n}(\lambda)=\left\{\tilde{f}_{i_{1}} \cdots \tilde{f}_{i_{r}} u_{\lambda}+q L_{n}(\lambda) \in L_{n}(\lambda) / q L_{n}(\lambda) \mid r \geq 0 \text { and } i_{k} \in I_{n}\right\} \backslash\{0\} .
$$

Lemma 4.1.1. Consider a vector $v \in V_{n}(\lambda)$ of weight $\delta$ such that $\operatorname{dim} V_{n}(\lambda)_{\delta}=1$. Then there exists a unique crystal basis $\left(L_{n}(\lambda), B_{n}(\lambda)\right)$ of $V_{n}(\lambda)$ such that $v \in L_{n}(\lambda)$ and $v+q L_{n}(\lambda) \in B_{n}(\lambda)$.

Proof. Let $u_{\lambda}$ be a highest weight vector of $V_{n}(\lambda)$ and $\left(L_{n}\left(u_{\lambda}\right), B_{n}\left(u_{\lambda}\right)\right)$ be the crystal basis corresponding to $u_{\lambda}$ by (10). Since $\operatorname{dim} V_{n}(\lambda)_{\delta}=1$, there exists a unique vertex $\widetilde{v}+q L_{n}\left(u_{\lambda}\right)$ of weight $\delta$ in the crystal $B_{n}\left(u_{\lambda}\right)$. Moreover we must have $\widetilde{v}=K(q) v$ with $K(q) \in \mathbb{Q}(q)$. Then $v_{\lambda}=\frac{1}{K(q)} u_{\lambda}$ is a highest weight vector in 
$V_{n}(\lambda)$ and the crystal basis $\left(L_{n}(\lambda), B_{n}(\lambda)\right)$ obtained from $v_{\lambda}$ as in (10) is such that $v \in L_{n}(\lambda)$ and $v+q L_{n}(\lambda) \in B_{n}(\lambda)$. This shows the "existence" part of the lemma.

Now suppose that $v_{\lambda}$ and $v_{\lambda}^{\prime}$ are two highest weight vectors in $V_{n}(\lambda)$ defining two crystal bases $\left(L_{n}(\lambda), B_{n}(\lambda)\right)$ and $\left(L_{n}^{\prime}(\lambda), B_{n}^{\prime}(\lambda)\right)$ verifying the assertion of the lemma. Set $v_{\lambda}^{\prime}=K(q) v_{\lambda}$ with $K(q) \in \mathbb{Q}(q)$. Let $\widetilde{f}=\widetilde{f}_{i_{1}} \cdots \widetilde{f}_{i_{r}}$ be a path in $B_{n}(\lambda)$ joining the highest weight vertex $b_{\lambda}=v_{\lambda}+q L_{n}(\lambda)$ to $v+q L_{n}(\lambda)$. Then $\widetilde{f}\left(v_{\lambda}\right) \neq 0$ in $V_{n}(\lambda)$ and has weight $\delta$. Write $\tilde{f}\left(v_{\lambda}\right)=F(q) v$ with $F(q) \in \mathbb{Q}(q)$. We have $\widetilde{f}\left(v_{\lambda}\right) \in L_{n}(\lambda), v \in L_{n}(\lambda)$ and $v \notin q L_{n}(\lambda)$ (otherwise $v+q L_{n}(\lambda)=0$ ). This implies that $F(q) \in \mathbf{A}(q)$. Moreover, we must have $\widetilde{f}\left(v_{\lambda}\right) \equiv v \bmod q L_{n}(\lambda)$. Thus $F(0)=1$. Similarly, we can write $\widetilde{f}\left(v_{\lambda}^{\prime}\right)=F^{\prime}(q) v$ with $F^{\prime}(q) \in \mathbf{A}(q)$ such that $F^{\prime}(0)=1$. By using the equality $v_{\lambda}^{\prime}=K(q) v_{\lambda}$, we derive $\widetilde{f}\left(v_{\lambda}^{\prime}\right)=F^{\prime}(q) v=K(q) F(q) v$. Hence $K(q)=\frac{F^{\prime}(q)}{F(q)} \in \mathbf{A}(q)$ and is such that $K(0)=1$. By Lemma 2.3.1, this implies that the crystal bases $\left(L_{n}(\lambda), B_{n}(\lambda)\right)$ and $\left(L_{n}^{\prime}(\lambda), B_{n}^{\prime}(\lambda)\right)$ coincide.

Definition 4.1.2. Consider a $U_{q}\left(\mathfrak{g}_{\infty}\right)$-module $M \in \mathcal{M}$ and denote by $m$ the minimal positive integer such that $M_{m} \neq\{0\}$. A crystal basis for $M$ is a pair $\left(L_{\infty}, B_{\infty}\right)=\left(L_{n}, B_{n}\right)_{n \geq m}$ verifying the following conditions:

(1) for any $n \geq 1,\left(L_{n}, B_{n}\right)$ is a crystal basis of $M_{n}$;

(2) for any $n \geq 1$, we have $L_{n} \subset L_{n+1}$ and $b+q L_{n} \in B_{n} \Longrightarrow b+q L_{n+1} \in B_{n+1}$;

(3) $L_{\infty}=\bigcup_{n \geq m} L_{n}$ and $B_{\infty}=\left\{b+q L_{\infty} \in L_{\infty} / q L_{\infty} \mid \exists n \geq m, b+q L_{n} \in B_{n}\right\}$.

Theorem 4.1.3. Consider $\lambda \in \mathcal{P}_{m}$ and let $v_{\lambda}^{(m)}$ be a fixed vector of highest weight $\phi_{m}(\lambda)$ in $V_{m}(\lambda)$. Then $V_{\infty}(\lambda)$ contains a unique crystal basis $\left(L_{\infty}(\lambda), B_{\infty}(\lambda)\right)=$ $\left(L_{n}(\lambda), B_{n}(\lambda)\right)_{n \geq m}$ such that for any $n \geq m, v_{\lambda}^{(m)} \in L_{n}(\lambda)$ and $v_{\lambda}^{(m)}+q L_{n}(\lambda) \in$ $B_{n}(\lambda)$.

Proof. By the above lemma, for any integer $n \geq m$, there exists a unique crystal basis $\left(L_{n}(\lambda), B_{n}(\lambda)\right)$ of $V_{n}(\lambda)$ such that $v_{\lambda}^{(m)} \in L_{n}(\lambda)$ and $v_{\lambda}^{(m)}+q L_{n}(\lambda) \in B_{n}(\lambda)$. We are first going to establish that $\left(L_{\infty}(\lambda), B_{\infty}(\lambda)\right)=\left(L_{n}(\lambda), B_{n}(\lambda)\right)_{n \geq m}$ is a crystal basis of $V_{\infty}(\lambda)$ in the sense of Definition 4.1.2.

Consider an integer $n \geq m$. Set $L_{n}^{\prime}(\lambda)=L_{n+1}(\lambda) \cap V_{n}(\lambda)$ and

$$
B_{n}^{\prime}(\lambda)=\left\{b+q L_{n}^{\prime}(\lambda) \mid b+q L_{n+1}(\lambda) \in B_{n+1}(\lambda), b \in L_{n}^{\prime}(\lambda)\right\} .
$$

Then $\left(L_{n}^{\prime}(\lambda), B_{n}^{\prime}(\lambda)\right)$ is a crystal basis of $V_{n}(\lambda)$ such that $v_{\lambda}^{(m)} \in L_{n}^{\prime}(\lambda)$ and $v_{\lambda}^{(m)}+q L_{n}^{\prime}(\lambda) \in B_{n}^{\prime}(\lambda)$. By the previous lemma, we must have $\left(L_{n}^{\prime}(\lambda), B_{n}^{\prime}(\lambda)\right)=$ $\left(L_{n}(\lambda), B_{n}(\lambda)\right)$. Thus $L_{n}(\lambda) \subset L_{n+1}(\lambda)$ and $b+q L_{n}(\lambda) \in B_{n}(\lambda) \Longrightarrow b+q L_{n+1}(\lambda) \in$ $B_{n+1}(\lambda)$. This shows that $\left(L_{\infty}(\lambda), B_{\infty}(\lambda)\right)=\left(L_{n}(\lambda), B_{n}(\lambda)\right)_{n \geq m}$ is a crystal basis of $V_{\infty}(\lambda)$.

Now if $\left(L_{\infty}(\lambda), B_{\infty}(\lambda)\right)=\left(L_{n}(\lambda), B_{n}(\lambda)\right)_{n \geq m}$ and $\left(L_{\infty}^{\prime}(\lambda), B_{\infty}^{\prime}(\lambda)\right)=$ $\left(L_{n}^{\prime}(\lambda), B_{n}^{\prime}(\lambda)\right)_{n \geq m}$ are two crystal bases of $V_{\infty}(\lambda)$ verifying the assertion of the theorem, we deduce immediately from Lemma 4.1.1 that for any $n \geq m$, the crystal bases $\left(L_{n}(\lambda), B_{n}(\lambda)\right)$ and $\left(L_{n}^{\prime}(\lambda), B_{n}^{\prime}(\lambda)\right)$ coincide.

Remarks. (i): If $\left(L_{\infty}, B_{\infty}\right)$ is a crystal basis of $M, L_{\infty}$ is a crystal lattice for $M$, and $B$ is a $\mathbb{Q}$ basis of $L_{\infty} / q L_{\infty}$ compatible with the weight decomposition of $M$ and stable under the action of the Kashiwara operators (see 8] Definition 4.2.3), then unfortunately, these conditions are not sufficient to guarantee the unicity of the crystal basis of $V_{\infty}(\lambda)$ because this module has no highest weight vector. 
(ii): The crystal basis $\left(L_{\infty}(\lambda), B_{\infty}(\lambda)\right)=\left(L_{n}(\lambda), B_{n}(\lambda)\right)_{n \geq m}$ of Theorem 4.1 .3 verifies

$$
L_{n}(\lambda)=L_{\infty}(\lambda) \cap V_{n}(\lambda)
$$

and

$$
B_{n}(\lambda)=\left\{v+q L_{n}(\lambda) \mid v+q L_{\infty}(\lambda) \in B_{\infty}(\lambda), v \in L_{n}(\lambda)\right\} .
$$

For any positive integer $p$, the crystal basis of $V_{\infty}\left(1^{p}\right)$ can be explicitly described by using the $q$-wedge product realization given in 3.3 . Consider the $\mathbf{A}(q)$-lattice $L_{\infty}^{(p)}=\bigoplus_{C \in \mathbf{C}(p)} \mathbf{A}(q) v_{C}$ in $V_{\infty}\left(1^{p}\right)$ and set $B_{\infty}^{(p)}=\left\{v_{C}+q L^{(p)} \mid C \in \mathbf{C}(p)\right\}$.

Proposition 4.1.4. The crystal basis $\left(L_{\infty}\left(1^{p}\right), B_{\infty}\left(1^{p}\right)\right)$ of $V_{\infty}\left(1^{p}\right)$ associated to the highest weight vector $v^{(p)}=v_{\bar{p}} \wedge v_{\overline{p-1}} \wedge \cdots \wedge v_{\overline{1}} \in V_{p}\left(1^{p}\right)$ by Theorem 10 verifies $L_{\infty}\left(1^{p}\right)=L_{\infty}^{(p)}$ and $B_{\infty}\left(1^{p}\right)=B_{\infty}^{(p)}$.

Proof. For any integer $n \geq 1$, one verifies (see Lemma 3.1.3 in [17) that $L_{n}\left(\left(1^{p}\right)\right)=$ $L_{\infty}^{(p)} \cap V_{n}\left(1^{p}\right)$ and $B_{n}\left(1^{p}\right)=\left\{v+q L_{n}\left(1^{p}\right) \mid v+q L^{(p)} \in B_{\infty}^{(p)}\right\}$ yields a crystal basis of $V_{n}\left(1^{p}\right)$ such that $v^{(p)} \in L_{n}\left(1^{p}\right)$ and $v^{(p)}+q L_{n}\left(1^{p}\right) \in B_{\infty}^{(p)}$. Moreover $L_{n}\left(1^{p}\right) \subset$ $L_{n+1}\left(1^{p}\right)$ and $b+q L_{n}\left(1^{p}\right) \in B_{n}(\lambda) \Longrightarrow b+q L_{n+1}\left(1^{p}\right) \in B_{n+1}\left(1^{p}\right)$. Thus by unicity of the crystal basis established in Theorem 4.1.3, $L_{\infty}\left(1^{p}\right)=\bigcup_{n \geq p} L_{n}\left(1^{p}\right)=L_{\infty}^{(p)}$ and $B_{\infty}\left(1^{p}\right)=B_{\infty}^{(p)}$.

Proposition 4.1.5. Suppose that $(L, B)$ and $\left(L^{\prime}, B^{\prime}\right)$ are crystal bases of the $U_{q}\left(\mathfrak{g}_{\infty}\right)$-modules $M$ and $M^{\prime}$ belonging to $\mathcal{M}$. Then

(1) $\left(L \oplus L^{\prime}, B \cup B^{\prime}\right)$ is a crystal basis of $M \oplus M^{\prime}$;

(2) $\left(L \otimes L^{\prime}, B \otimes B^{\prime}\right)$ with $B \otimes B^{\prime}=\left\{b \otimes b^{\prime} \mid b \in B, b^{\prime} \in B^{\prime}\right\}$ is a crystal basis of $M \otimes M^{\prime}$, where the action of the Kashiwara operators $\widetilde{e}_{i}$ and $\widetilde{f}_{i}, i \in I$, on $B \otimes B^{\prime}$ is given by (7) and (8).

Proof. The proof of (1) is the same as in the finite rank case (see Theorem 4.2.10 in $[8]$ ).

For (2), consider the sequence $\left(L_{n}^{\otimes}, B_{n}^{\otimes}\right)_{n \geq m}$ where $L_{n}^{\otimes}=\left(L \otimes L^{\prime}\right) \cap\left(M \otimes M^{\prime}\right)_{n}$ and

$$
B_{n}^{\otimes}=\left\{\left(v \otimes v^{\prime}+q L_{n}^{\otimes} \mid v \otimes v^{\prime} \in L_{n}^{\otimes},(v+q L) \otimes\left(v+q L^{\prime}\right) \in B \otimes B^{\prime}\right\} .\right.
$$

Each pair $\left(L_{n}^{\otimes}, B_{n}^{\otimes}\right)$ is a crystal basis of $\left(M \otimes M^{\prime}\right)_{n}$ and the sequence $\left(L_{n}^{\otimes}, B_{n}^{\otimes}\right)_{n \geq m}$ verifies the conditions of Definition 4.1.2, For any integer $n$, the action of the Kashiwara operators on $B_{n}^{\otimes}$ is given by (77) and (8). We have $L \otimes L^{\prime}=\bigcup_{n \geq m} L_{n}^{\otimes}$ and

$$
B \otimes B^{\prime}=\left\{v \otimes v^{\prime}+q L \otimes L^{\prime} \mid \exists n \geq m, v \otimes v^{\prime}+q L_{n}^{\otimes} \in B_{n}^{\otimes}\right\} .
$$

Moreover, the conditions $L_{n}^{\otimes} \subset L_{n+1}^{\otimes}$ and $v+q L_{n}^{\otimes} \in B_{n} \Longrightarrow v+q L_{n+1}^{\otimes} \in B_{n+1}$ imply that the action of the Kashiwara operators $\widetilde{e}_{i}, \widetilde{f}_{i}$ with $i \in I_{n}$ on $\left(L_{n}, B_{n}\right)$ and $\left(L_{n+1}, B_{n+1}\right)$ coincide. Thus the action of these operators on $B \otimes B^{\prime}$ is also given by rules (7) and (8).

Consider a $U_{q}\left(\mathfrak{g}_{\infty}\right)$-module $M$ belonging to $\mathcal{M}$ with crystal basis $(L, B)$. Write $M=\bigoplus V_{\infty}^{(k)}$ for its decomposition into irreducible components. For any $k$, let $\left(L^{(k)}, B^{(k)}\right)$ be the crystal basis of the irreducible module $V_{\infty}^{(k)}$. By Proposition 4.1.5, we know that $\left(\bigoplus L^{(k)}, \bigcup B^{(k)}\right)$ is also a crystal basis of $M$. 
Theorem 4.1.6. There exists an isomorphism of crystal bases

$$
\psi:(L, B) \stackrel{\simeq}{\rightarrow}\left(\bigoplus L^{(k)}, \bigcup B^{(k)}\right) .
$$

Proof. We write for short $V_{n}^{(k)}$ for $\left(V_{\infty}^{(k)}\right)_{n}$. We have $M=\bigoplus V_{\infty}^{(k)}$; thus for any integer $n \geq 1, M_{n}=\bigoplus V_{n}^{(k)}$. Set $L_{n}=L \cap M_{n}$ and $B_{n}=\left\{v+q L_{n} \mid v \in L_{n}, v+q L \in\right.$ $B\}$. Then $\left(L_{n}, B_{n}\right)$ is a crystal basis of $M_{n}$. Similarly for any $k, L_{n}^{(k)}=L^{(k)} \cap V_{n}^{(k)}$ and $B_{n}^{(k)}=\left\{v+q L_{n}^{(k)} \mid v \in L_{n}^{(k)}, v+q L^{(k)} \in B^{(k)}\right\}$ define a crystal basis of $V_{n}^{(k)}$. Thus $\left(\bigoplus L_{n}^{(k)}, \bigcup B_{n}^{(k)}\right)$ is also a crystal basis of $M_{n}$. This implies that for any integer $n \geq 1$, there exists an isomorphism of crystal bases

$$
\psi_{n}:\left(L_{n}, B_{n}\right) \stackrel{\simeq}{\rightarrow}\left(\bigoplus L_{n}^{(k)}, \bigcup B_{n}^{(k)}\right) .
$$

The conditions

$$
\left\{\begin{array}{l}
L_{n}=L_{n+1} \cap M_{n}, B_{n}=B_{n+1} \cap\left(L_{n} / q L_{n}\right) \\
L_{n}^{(k)}=L_{n+1}^{(k)} \cap V_{n}^{(k)}, B_{n}^{(k)}=B_{n+1}^{(k)} \cap\left(L_{n}^{(k)} / q L_{n}^{(k)}\right)
\end{array} \quad \text { for any } n \geq 1\right.
$$

show that the restriction of $\psi_{n+1}$ to $\left(L_{n}, B_{n}\right)$ is also an isomorphism of crystal bases from $\left(L_{n}, B_{n}\right)$ to $\left(\bigoplus L_{n}^{(k)}, \bigcup B_{n}^{(k)}\right)$. Indeed, $\psi_{n+1}\left(L_{n}\right)=\bigoplus L_{n+1}^{(k)} \cap M_{n}=\bigoplus L_{n}^{(k)}$ for $L_{n}=L_{n+1} \cap M_{n}$ and $L_{n}^{(k)}=L_{n+1}^{(k)} \cap V_{n}^{(k)}$. Similarly

$\psi_{n+1}\left(B_{n}\right)=\left(\bigcup B_{n+1}^{(k)}\right) \cap\left(\bigoplus L_{n}^{(k)} / \bigoplus q L_{n}^{(k)}\right)=\bigcup\left(B_{n+1}^{(k)} \cap L_{n}^{(k)} / q L_{n}^{(k)}\right)=\bigcup B_{n}^{(k)}$.

This permits us to choose the isomorphisms $\psi_{n}$ so that for any integer $n, \psi_{n}$ is the restriction of $\psi_{n+1}$ to $\left(L_{n}, B_{n}\right)$. Then one defines

$$
\psi:(L, B) \rightarrow\left(\bigoplus L^{(k)}, \bigcup B^{(k)}\right)
$$

by requiring that $\psi(v)=\psi_{n}(v)$ for any $v \in L_{n}$. The map $\psi: L \rightarrow \bigoplus L^{(k)}$ is an A $(q)$-linear isomorphism which commutes with the Kashiwara operators. Moreover, the induced $\mathbb{Q}$-linear isomorphism from $L / q L$ to $\bigoplus L^{(k)} / q \oplus L^{(k)}$ defines an isomorphism of crystals from $B$ to $\bigcup B^{(k)}$. Thus $\psi$ is an isomorphism of crystal bases.

Remark. We deduce from the previous theorem that the decomposition of $M$ into its irreducible components is given by the decomposition of $B$ into its connected components.

4.2. Canonical basis for the modules $V_{\infty}(\lambda)$. Denote by $F \mapsto \bar{F}$ the involution of $U_{q}\left(\mathfrak{g}_{\infty}\right)$ defined as the ring automorphism satisfying

$$
\bar{q}=q^{-1}, \quad t_{i}=t_{i}^{-1}, \quad \overline{e_{i}}=e_{i}, \quad \overline{f_{i}}=f_{i} \quad \text { for } i \in I .
$$

Let $U_{\mathbb{Q}}^{-}$(resp. $U_{n, \mathbb{Q}}^{-}$) be the subalgebra of $U_{q}\left(\mathfrak{g}_{\infty}\right)$ generated over $\mathbb{Q}\left[q, q^{-1}\right]$ by the $f_{i}^{(k)}, i \in I$ (resp. $i \in I_{n}$ ). Let us first recall the definition of the canonical basis of $V_{n}(\lambda)$. Let $v_{\lambda}^{n} \in V_{n}(\lambda)$ be a highest weight vector of weight $\phi_{n}(\lambda)$. By writing each vector $v$ of $V_{n}(\lambda)$ in the form $v=F v_{\lambda}^{(n)}$ where $F \in U_{q}^{-}\left(\mathfrak{g}_{n}\right)$, we obtain an involution $I_{n}$ of $V_{n}(\lambda)$ defined by

$$
I_{n}(v)=\bar{F} v_{\lambda}^{(n)} .
$$

Set $V_{n, \mathbb{Q}}(\lambda)=U_{n, \mathbb{Q}}^{-} v_{\lambda}^{(n)}$ and denote by $\left(L_{n}(\lambda), B_{n}(\lambda)\right)$ the crystal basis of $V_{n}(\lambda)$ determined by $v_{\lambda}^{(n)}$. 
Theorem 4.2.1 (Kashiwara). There exists a unique $\mathbb{Q}\left[q, q^{-1}\right]$-basis $G_{n}(\lambda)=$ $\left\{G_{n}(b) \mid b \in B_{n}(\lambda)\right\}$ of $V_{n, \mathbb{Q}}(\lambda)$ such that:

$$
\begin{gathered}
G_{n}(b) \equiv b \bmod q L_{n}(\lambda), \\
I_{n}\left(G_{n}(b)\right)=G_{n}(b) .
\end{gathered}
$$

The basis $G_{n}(\lambda)$ is called the canonical basis of $V_{n}(\lambda)$. It is determined uniquely up to the choice of a highest weight vector in $V_{n}(\lambda)$.

Remark. Suppose that the highest weight vector $v_{\lambda}^{(n)}$ is replaced by $\left(v_{\lambda}^{(n)}\right)^{\prime}=$ $K(q) v_{\lambda}^{(n)}$, where $K(q) \in \mathbb{Q}(q)$. Then the canonical basis $G_{n}^{\prime}(\lambda)$ defined from $\left(v_{\lambda}^{(n)}\right)^{\prime}$ verifies $G_{n}^{\prime}(\lambda)=K(q) G_{n}(b)$. Moreover, we have $I^{\prime}(v)=\frac{K(q)}{K\left(q^{-1}\right)} I(v)$, where $I_{n}^{\prime}$ is the involution corresponding to $\left(v_{\lambda}^{(n)}\right)^{\prime}$.

Lemma 4.2.2. Consider a vector $v \in V_{n}(\lambda)$ of weight $\delta$ such that $\operatorname{dim} V_{n}(\lambda)_{\delta}=1$. Then there exists a unique highest weight vector $v_{\lambda}^{(n)} \in V_{n}(\lambda)$ such that $v$ is a vector of the canonical basis $G_{n}(\lambda)$ obtained from $v_{\lambda}^{(n)}$.

Proof. Let $u_{\lambda}$ be a highest weight vector of $V_{n}(\lambda)$. Write $G_{n}^{u_{\lambda}}(\delta)$ for the unique vector of weight $\delta$ in the canonical basis determined by $u_{\lambda}$. We must have $G_{n}^{u_{\lambda}}(\delta)=$ $K(q) v$, where $K(q) \in \mathbb{Q}(q)$ for $\operatorname{dim} V_{n}(\lambda)_{\delta}=1$. Set $v_{\lambda}^{(n)}=\frac{1}{K(q)} u_{\lambda}$. The vector of weight $\delta$ in the canonical basis determined by $v_{\lambda}^{(n)}$ is equal to $\frac{1}{K(q)} G_{n}^{u_{\lambda}}(\delta)=v$ (see remark above) as required. This shows the "existence" part of the lemma.

Now suppose that $v_{\lambda}^{(n)}$ and $\left(v_{\lambda}^{(n)}\right)^{\prime}$ are two highest weight vectors in $V_{n}(\lambda)$ such that $v$ belongs to the canonical bases $G_{n}(\lambda)$ and $G_{n}^{\prime}(\lambda)$ determined respectively by $v_{\lambda}^{(n)}$ and $\left(v_{\lambda}^{(n)}\right)^{\prime}$. Write $\left(v_{\lambda}^{(n)}\right)^{\prime}=K(q) v_{\lambda}^{(n)}$, where $K(q) \in \mathbb{Q}(q)$. Denote by $G_{n}(\delta)$ and $G_{n}^{\prime}(\delta)$ the vectors of weight $\delta$ respectively in the canonical bases $G_{n}(\lambda)$ and $G_{n}^{\prime}(\lambda)$. We must have $G_{n}^{\prime}(\delta)=K(q) G_{n}(\delta)$. Since $\operatorname{dim} V_{n}(\lambda)_{\delta}=1$ and $v$ belongs to $G_{n}(\lambda) \cap G_{n}^{\prime}(\lambda)$, we obtain $G_{n}(\delta)=G_{n}^{\prime}(\delta)=v$. Thus $K(q)=1$ and $v_{\lambda}^{(n)}=$ $\left(v_{\lambda}^{(n)}\right)^{\prime}$

The canonical basis $G_{n+1}(\lambda)$ defined by $v_{\lambda}^{(n+1)}$ (with the notation of the above proof) contains a unique vector $u_{\lambda}^{(n)}$ of weight $\phi_{n}(\lambda)$. This vector is of highest weight in $V_{n}(\lambda)$. Denote by $G_{n}^{(n+1)}(\lambda)$ the canonical basis of $V_{n}(\lambda)$ defined from $u_{\lambda}^{(n)}$. Write $I_{n}^{(n+1)}$ for the corresponding involution defined on $V_{n}(\lambda)$ and set $V_{n, \mathbb{Q}}^{(n+1)}(\lambda)=$ $U_{n, \mathbb{Q}}^{-} u_{\lambda}^{(n)}$.

Lemma 4.2.3. For any $v \in V_{n}(\lambda)$, we have $I_{n}^{(n+1)}(v)=I_{n+1}(v)$. Moreover $V_{n, \mathbb{Q}}^{(n+1)}(\lambda) \subset V_{n+1, \mathbb{Q}}(\lambda)$ and $G_{n}^{(n+1)}(\lambda) \subset G_{n+1}(\lambda)$.

Proof. The vector $u_{\lambda}^{(n)}$ belongs to $V_{n+1}(\lambda)$; thus there exists $R \in U_{q}\left(\mathfrak{g}_{n+1}\right)$ such that $u_{\lambda}^{(n)}=R \cdot v_{\lambda}^{(n+1)}$. Consider $v \in V_{n}(\lambda)$ and set $v=F \cdot v_{\lambda}^{n}$ where $F \in U_{q}\left(\mathfrak{g}_{n}\right)$. Then $v=F R \cdot v_{\lambda}^{(n+1)}$. We have $I_{n+1}(v)=\overline{F R} \cdot v_{\lambda}^{(n+1)}=\bar{F}\left(\bar{R} \cdot v_{\lambda}^{(n+1)}\right)$. Since $u_{\lambda}^{(n)} \in G_{n+1}(\lambda)$, we must have $I_{n+1}\left(u_{\lambda}^{(n)}\right)=u_{\lambda}^{(n)}=\bar{R} \cdot v_{\lambda}^{(n+1)}$. This gives $I_{n+1}(v)=$ $\bar{F} \cdot u_{\lambda}^{(n)}=I_{n}^{(n+1)}(v)$. The inclusion $V_{n \mathbb{Q}}^{(n+1)}(\lambda) \subset V_{n+1, \mathbb{Q}}(\lambda)$ is immediate. We deduce that the vectors $G_{n}^{(n+1)}(b)$ of $G_{n}^{(n+1)}(\lambda)$ belong to $V_{n+1, \mathbb{Q}}(\lambda)$. Moreover 
they verify $G_{n}^{(n+1)}(b) \equiv b \bmod q L_{n+1}(\lambda)$ and $I_{n+1}\left(G_{n}^{(n+1)}(b)\right)=G_{n}^{(n+1)}(b)$. Thus $G_{n}^{(n+1)}(\lambda) \subset G_{n+1}(\lambda)$.

Now suppose that $\lambda \in \mathcal{P}_{m}$ and fix a vector $v_{\lambda}^{(m)}$ in $V_{m}(\lambda)$ of highest weight $\phi_{m}(\lambda)$. For any $n \geq m, v_{\lambda}^{(m)}$ belongs to $V_{n}(\lambda)$ and $\operatorname{dim} V_{n}(\lambda)_{\phi_{m}(\lambda)}=1$. Thus by the above lemma, there is a unique highest weight vector $v_{\lambda}^{(n)} \in V_{n}(\lambda)$ such that $v_{\lambda}^{(m)}$ belongs to the canonical basis $G_{n}(\lambda)$ determined by $v_{\lambda}^{(n)}$. This yields an involution $I_{n}$ on $V_{n}(\lambda)$ and a subspace $V_{n, \mathbb{Q}}(\lambda)$ which depends only on the choice of $v_{\lambda}^{(m)}$.

For any integer $n$, the family of canonical bases $\left(G_{n}(\lambda)\right)_{n \geq 1}$ determined by the condition $v_{\lambda}^{(m)} \in G_{n}(\lambda)$ verifies $G_{n}(\lambda) \subset G_{n+1}(\lambda)$. Indeed, $G_{n+1}(\lambda)$ contains a unique vector $u_{\lambda}^{(n)}$ of weight $\phi_{n}(\lambda)$. This vector is of highest weight in $V_{n}(\lambda)$. By Lemma 4.2.3, we obtain that $v_{\lambda}^{m}$ belongs to the canonical basis of $V_{n}(\lambda)$ determined by $u_{\lambda}^{(n)}$. Thus by Lemma 4.2.2 we have $u_{\lambda}^{(n)}=v_{\lambda}^{(n)}$. In particular for any $n \geq m$, $I_{n}$ is the restriction of $I_{n+1}$ on $V_{n}(\lambda)$. This permits us to define the bar involution on $V_{\infty}(\lambda)$ by

$$
\bar{v}=I_{n}(v) \text { for any } v \in V_{n}(\lambda) .
$$

Set $V_{\mathbb{Q}}(\lambda)=\bigcup_{n \geq m} V_{n, \mathbb{Q}}(\lambda)$ and consider the crystal basis $\left(L_{\infty}(\lambda), B_{\infty}(\lambda)\right)$ of $V_{\infty}(\lambda)$ determined by $v_{\lambda}^{(m)}$ as in Theorem 4.1.3. The following theorem follows immediately from the previous arguments.

Theorem 4.2.4. Consider $\lambda \in \mathcal{P}_{m}$ and $v_{\lambda}^{(m)}$ a highest weight vector of $V_{m}(\lambda)$. There exists a unique $\mathbb{Q}\left[q, q^{-1}\right]$-basis $G_{\infty}(\lambda)=\left\{G_{\infty}(b) \mid b \in B_{\infty}(T)\right\}$ of $V_{\mathbb{Q}}(\lambda)$ such that:

$$
\begin{gathered}
v_{\lambda}^{(m)} \in G_{\infty}(\lambda), \\
G_{\infty}(b) \equiv b \bmod q L_{\infty}(\lambda), \\
\overline{G_{\infty}(b)}=G_{\infty}(b) .
\end{gathered}
$$

We will call $G_{\infty}(\lambda)$ the canonical basis of $V_{\infty}(\lambda)$. It is determined uniquely up to the choice of a highest weight vector in $V_{m}(\lambda)$.

Remark. One has $G_{\infty}(\lambda)=\bigcup_{n \geq m} G_{n}(\lambda)$, where $G_{n}(\lambda)$ is the canonical basis of $V_{n}(\lambda)$ determined by $v_{\lambda}^{(m)}$.

\section{ROBINSON-SCHENSTED CORRESPONDENCES AND BI-CRYSTALS}

5.1. Insertion schemes. We have seen in $\$ 3.2$ that the vertices of $B_{n}(1)$ are labelled by the letters of the alphabet $\mathcal{X}_{n}$. This permits us to identify the vertices of the crystal graph $G_{n}=\bigoplus_{l \geq 0} B_{n}(1)^{\otimes l}$ with the words on $\mathcal{X}_{n}$. For any $w \in G_{n}$ we have $\operatorname{wt}(w)=\left(d_{1}, \ldots, d_{n}\right) \in P_{n}$, where for all $i=1, \ldots, n, d_{i}$ is the number of letters $\bar{i}$ of $w$ minus its number of letters $i$.

Consider $\lambda \in \mathcal{P}_{m}$ with $m \leq n$ and denote by $Y(\lambda)$ the Young diagram of shape $\lambda$. Write $T_{n, \lambda}$ for the filling of $Y(\lambda)$ whose $k$-th row contains only letters $\overline{n-k+1}$. Let $b_{n, \lambda}$ be the vertex of $B_{n}(1)^{\otimes|\lambda|}$ obtained by column reading $T_{n, \lambda}$, that is, by reading $T_{n, \lambda}$ from right to left and top to bottom. Kashiwara and Nakashima realize $B_{n}(\lambda)$ as the connected component of the tensor power $B_{n}(1)^{\otimes|\lambda|}$ of the highest weight vertex $b_{n, \lambda}$. The Kashiwara-Nakashima tableaux of type $A_{n-1}, B_{n}, C_{n}, D_{n}$ 
and shape $\lambda$ are defined as the fillings of $Y(\lambda)$ having a column reading which belongs to $B_{n}(\lambda)$ (recall that $B_{n}(\lambda)$ depends on the root system considered). In the sequel we will denote by $\mathrm{w}(T)$ the column reading of the tableau $T$. When there is no risk of confusion, we sometimes identify $T$ with $\mathrm{w}(T)$.

Write $\mathbf{T}_{n}(\lambda)$ respectively for the sets of Kashiwara-Nakashima tableaux of shape $\lambda$. Set $\mathbf{T}_{n}=\bigcup_{\lambda \in \mathcal{P}_{m}, m \leq n} \mathbf{T}_{n}(\lambda)$ and $\mathbf{T}=\bigcup_{n \geq 1} \mathbf{T}_{n}$. In the sequel we only summarize the combinatorial description of $\mathbf{T}_{n}(\lambda)$ and refer the reader to [12, 15] and [16] for more details.

When $\lambda$ is a column partition, the tableaux of $\mathbf{T}_{n}(\lambda)$ are called $n$-admissible columns. The $n$-admissible columns of types $A_{n-1}, B_{n}, C_{n}$ and $D_{n}$ are in particular columns of types $A_{n-1}, B_{n}, C_{n}$ and $D_{n}$, that is, columns of types $A_{\infty}, B_{\infty}, C_{\infty}$ and $D_{\infty}$ (see (20) $)$ with letters in $\mathcal{X}_{n}$. For type $A_{n-1}$, each column is $n$-admissible. This is not true for the types $B_{n}, C_{n}$ and $D_{n}$. More precisely a column $C$ of (20) is $n$ admissible if and only if it can be duplicated following a simple algorithm described in [16] into a pair $(l C, r C)$ of columns without a pair of opposite letters $(x, \bar{x})$ (the letter 0 is counted as the pair $(0, \overline{0}))$ and containing only letters $a$ such that $a \in \mathcal{X}_{n}$.

Example 5.1.1. For the column $C=$\begin{tabular}{|l|}
\hline$\overline{3}$ \\
\hline$\overline{1}$ \\
\hline 0 \\
\hline 1 \\
\hline 2 \\
\hline
\end{tabular}

$$
l C=\begin{array}{|l|}
\hline \overline{5} \\
\hline \overline{4} \\
\hline \overline{3} \\
\hline 1 \\
\hline 2 \\
\hline
\end{array} \text { and } r C=\begin{array}{|c|}
\hline \overline{3} \\
\hline \overline{1} \\
\hline 3 \\
\hline 4 \\
\hline 5 \\
\hline
\end{array}
$$

Hence $C$ is 5 -admissible but not $n$-admissible for $n \leq 4$.

For a general $\lambda$ a tableau $T \in \mathbf{T}_{n}(\lambda)$ can be regarded as a filling of the Young diagram of shape $\lambda$ such that

- $T=C_{1} \cdots C_{r}$, where the columns $C_{i}$ of $T$ are $n$-admissible,

- for any $i \in\{1, \ldots, r-1\}$ the columns of the tableau $r\left(C_{i}\right) l\left(C_{i+1}\right)$ weakly increase from left to right and do not contain special configurations (detailed in 12 and [16]) when $T$ is of type $D_{n}$.

Remarks. (i) : For each column $C$, there is an integer $n$ such that $C$ is not $i$ admissible for any $i<n$ but is $i$-admissible for each $i \geq n$.

(ii) : We have $\mathbf{T}_{n}(\lambda) \subset \mathbf{T}_{n+1}(\lambda)$ since the $n$-admissible columns are also $(n+1)$ admissible and the duplication process of a column does not depend on $n$.

(iii) : For type $A_{n-1}$, we have $l C=r C=C$ and $\mathbf{T}_{n}(\lambda)$ is the set of semistandard tableaux of shape $\lambda$.

There exist insertion schemes related to each classical root system [15], [16] and analogous for Kashiwara-Nakashima tableaux to the well-known bumping algorithm on semistandard tableaux.

Denote by $\sim_{n}$ the equivalence relation defined on the vertices of $G_{n}$ by $w_{1} \sim_{n} w_{2}$ if and only if $w_{1}$ and $w_{2}$ occur at the same place in two isomorphic connected components of $G_{n}$. For any word $w$, the insertion schemes permit us to compute 
the unique tableau $P_{n}(w)$ such that $w \sim_{n} \mathrm{w}\left(P_{n}(w)\right)$. In fact $\sim_{n}$ is a congruence $\equiv_{n}$ ([15], [16], [18]) which depends on the type considered. For type $A_{n-1}, \equiv_{n}$ is the congruence defined by the Knuth relations. For types $B_{n}, C_{n}$ and $D_{n}$, it is obtained as the quotient of the free monoid of words on $\mathcal{X}_{n}$ by two kinds of relations.

The first consists of relations of length 3 analogous to the Knuth relations. In fact these relations are precisely those which are needed to describe the insertion

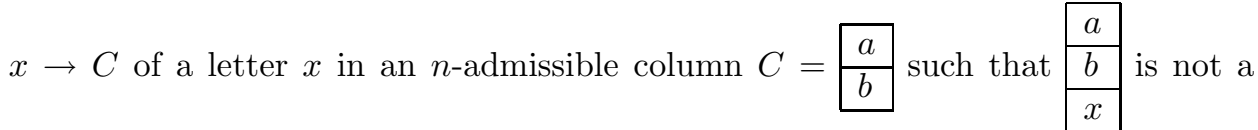
column. This can be written

$$
x \rightarrow \begin{array}{|l|l|}
\hline a & a \\
\hline b
\end{array}=\begin{array}{|l|l|}
\hline x & b \\
\hline a^{\prime} & x^{\prime} \\
\hline b^{\prime} &
\end{array}
$$

and, contrary to the insertion scheme for the semistandard tableaux, the sets $\left\{a^{\prime}, b^{\prime}, x^{\prime}\right\}$ and $\{a, b, c\}$ are not necessarily equal (i.e. the relations are not homogeneous in general).

Next we have the contraction relations which do not preserve the length of the words. These relations are precisely those which are needed to describe the insertion $x \rightarrow C$ of a letter $x$ such that $\bar{n} \leq x \leq n$ in an $n$-admissible column $C$ such that

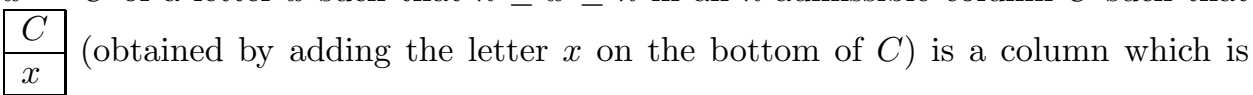
not $n$-admissible. In this case $\frac{C}{x}$ is necessarily $(n+1)$-admissible and has to be contracted to give an $n$-admissible column. We obtain $x \rightarrow C=\widetilde{C}$ with $\widetilde{C}$ an $n$-admissible column of height $h(C)$ or $h(C)-1$.

The insertion of the letter $x$ in an $n$-admissible column $C$ of arbitrary height

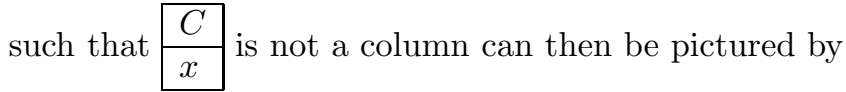

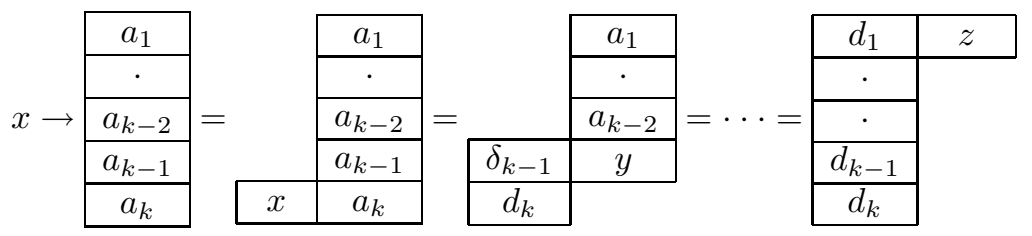

that is, one elementary transformation (25) is applied to each step. One proves that $x \rightarrow C$ is then a tableau of $\mathbf{T}^{(n)}$ with two columns respectively of height $h(C)$ and 1 .

Now we can define the insertion $x \rightarrow T$ of the letter $x$ such that $\bar{n} \leq x \leq n$ in the tableau $T \in \mathbf{T}_{n}(\lambda)$. Set $T=C_{1} \cdots C_{r}$ where $C_{i}, i=1, \ldots, r$ are the $n$-admissible columns of $T$.

(1) When \begin{tabular}{|l|l|l|l|}
\hline & $C_{1}$ \\
\hline$x$ & is not a column, write $x \rightarrow C=C_{1}^{\prime}$ & $y$ \\
\end{tabular} admissible column of height $h\left(C_{1}\right)$ and $y$ is a letter. Then $x \rightarrow T=C_{1}^{\prime}(y \rightarrow$ $\left.C_{2} \cdots C_{r}\right)$; that is, $x \rightarrow T$ is the juxtaposition of $C_{1}^{\prime}$ with the tableau $\widehat{T}$ obtained by inserting $y$ in the tableau $C_{2} \cdots C_{r}$.

(2) When $\frac{C_{1}}{x}$ is an $n$-admissible column, $x \rightarrow T$ is the tableau obtained by adding a box containing $x$ on the bottom of $C_{1}$. 
(3) When \begin{tabular}{ll}
\hline$C_{1}$ \\
\hline$x$
\end{tabular} is a column which is not $n$-admissible, write $x \rightarrow C=\widetilde{C}$ and set $\mathrm{w}(\widetilde{C})=y_{1} \cdots y_{s}$, where the $y_{i}$ 's are letters. Then $x \rightarrow T=y_{s} \rightarrow$ $\left(y_{s-1} \rightarrow\left(\cdots y_{1} \rightarrow \widehat{T}\right)\right)$; that is, $x \rightarrow T$ is obtained by inserting successively the letters of $\widetilde{C}$ into the tableau $\widehat{T}=C_{2} \cdots C_{r}$. Note that there is no new contraction during these $s$ insertions.

Remarks. (i): The $P_{n}$-symbol defined above can be computed recursively by setting $P_{n}(w)=w$ if $w$ is a letter and $P_{n}(w)=x \rightarrow P_{n}(u)$ where $w=u x$ with $u$ a word and $x$ a letter otherwise.

(ii): Consider $T \in \mathbf{T}_{n}(\lambda) \subset \mathbf{T}_{n+1}(\lambda)$ and a letter $x$ such that $\bar{n} \leq x \leq n$. The tableau obtained by inserting $x$ in $T$ may depend on whether $T$ is regarded as a tableau of $\mathbf{T}_{n}(\lambda)$ or as a tableau of $\mathbf{T}_{n+1}(\lambda)$. Indeed if \begin{tabular}{l|l}
\hline$C_{1}$ \\
\hline$x$
\end{tabular} is not $n$-admissible, then it is necessarily $(n+1)$-admissible since $C_{1}$ is $n$-admissible. Hence there is no contraction during the insertion $x \rightarrow T$ when it is regarded as a tableau of $\mathbf{T}_{n+1}(\lambda)$.

(iii): Consider $w \in \mathcal{X}_{n}$. From (ii) we deduce that there exists an integer $N \geq n$ minimal such that $P_{N}(w)$ can be computed without using the contraction relation. Then for any $k \geq N, P_{k}(w)=P_{N}(w)$.

(iv): Similarly to the bumping algorithm for semistandard tableaux, the insertion algorithms described above are reversible.

We give below the plactic relations of length 3 . The contraction relations will not be used in the sequel:

5.1.1. For type $A_{n-1}$.

$$
\left\{\begin{array}{l}
a b x=b a x \text { if } a<x \leq b \\
a b x=a x b \text { if } x \leq a<b
\end{array}\right.
$$

where $a, b, x$ are letters of $\mathcal{X}_{A_{n-1}}$.

\subsubsection{For type $B_{n}$.}

$$
\begin{gathered}
\left\{\begin{array}{l}
a b x \equiv b a x \text { if } a<x<b \text { and } b \neq \bar{a} \\
a b x \equiv a x b \text { if } x<a<b \text { and } b \neq \bar{x}
\end{array},\left\{\begin{array}{l}
a x x \equiv x a x \text { if } a<x, x \neq \bar{a} \text { and } x \neq 0 \\
x b x \equiv x x b \text { if } x<b, b \neq \bar{x} \text { and } x \neq 0
\end{array},\right.\right. \\
\left\{\begin{array}{l}
\bar{b} b x \equiv(b+1)(\overline{b+1}) x \text { if } \bar{b} \leq x \leq b \text { and } b \neq 0 \\
a b \bar{b} \equiv a(\overline{b-1})(b-1) \text { if } \bar{b}<a<b \text { and }(a, b) \neq(0,1)
\end{array}\right. \\
\left\{\begin{array}{l}
00 x \equiv 0 x 0 \text { if } x \leq \overline{1} \\
0 b 0 \equiv b 00 . \text { if } b \geq 1
\end{array}, \quad 01 \overline{1} \equiv 1 \overline{1} 0\right.
\end{gathered}
$$

where $a, b, x$ are letters of $\mathcal{X}_{B_{n}}$.

5.1.3. For type $C_{n}$.

$$
\left\{\begin{array} { l } 
{ a b x \equiv b a x \text { if } a < x \leq b \text { and } b \neq \overline { a } } \\
{ a b x \equiv a x b \text { if } x \leq a < b \text { and } b \neq \overline { x } }
\end{array} \text { and } \left\{\begin{array}{l}
\bar{b} b x \equiv(b+1)(\overline{b+1}) x \text { if } \bar{b} \leq x \leq b \\
a b \bar{b} \equiv a(\overline{b-1})(b-1) \text { if } \bar{b}<a<b
\end{array}\right.\right.
$$

where $a, b, x$ are letters of $\mathcal{X}_{C_{n}}$. 
5.1.4. For type $D_{n}$.

$$
\begin{gathered}
\left\{\begin{array}{l}
a b x \equiv b a x \text { if } a<x \leq b \text { and } b \neq \bar{a} \\
a b x \equiv a x b \text { if } x \leq a<b \text { and } b \neq \bar{x}
\end{array},\left\{\begin{array}{l}
\bar{b} b x \equiv(b+1)(\overline{b+1}) x \text { if } \bar{b} \leq x \leq b \\
a b \bar{b} \equiv a(\overline{b-1})(b-1) \text { if } \bar{b}<a<b
\end{array}\right.\right. \\
\left\{\begin{array}{l}
1 b \overline{1} \equiv b 1 \overline{1} \text { if } b \geq 2 \\
\overline{1} b 1 \equiv b \overline{1} 1 \text { if } b \geq 2
\end{array}, \quad\left\{\begin{array}{l}
1 \overline{1} x \equiv 1 x \overline{1} \text { if } x \leq \overline{2} \\
\overline{1} 1 x \equiv \overline{1} x 1 \text { if } x \leq \overline{2}
\end{array},\left\{\begin{array}{l}
\overline{1} 11 \equiv 2 \overline{2} 1 \\
1 \overline{1} \overline{1} \equiv 2 \overline{2} \overline{1}
\end{array}, \quad\left\{\begin{array}{l}
12 \overline{1} \equiv 11 \overline{1} \\
\overline{1} 21 \equiv \overline{1} \overline{1} 1
\end{array}\right.\right.\right.\right.
\end{gathered}
$$

where $a, b, x$ are letters of $\mathcal{X}_{D_{n}}$.

5.2. Plactic monoids for types $A_{\infty}, B_{\infty}, C_{\infty}$ and $D_{\infty}$. Consider $\lambda \in \mathcal{P}_{m}, v_{\lambda}^{(m)} \in$ $V_{m}(\lambda)$ a highest weight vector and $\left(L_{\infty}(\lambda), B_{\infty}(\lambda)\right)$ the crystal basis of $V_{\infty}(\lambda)$ determined by $v_{\lambda}^{(m)}$. By definition of the crystal basis $\left(L_{\infty}(\lambda), B_{\infty}(\lambda)\right)$, there is a natural embedding of crystals

$$
\iota_{n}:\left\{\begin{array}{l}
B_{n}(\lambda) \rightarrow B_{n+1}(\lambda), \\
b+q L_{n}(\lambda) \mapsto b+q L_{n+1}(\lambda) .
\end{array}\right.
$$

By identifying $B_{n}(\lambda)$ with its image under $\iota_{n}$, one can write $B_{\infty}(\lambda)=\bigcup_{n \geq m} B_{n}(\lambda)$.

Example 5.2.1. The crystal graph of the vector representation of $U_{q}\left(g_{B_{\infty}}\right)$ is identified with the infinite crystal

$B_{\infty}(1): \cdots \rightarrow \bar{n} \stackrel{n-1}{\rightarrow} \overline{n-1} \stackrel{n-2}{\rightarrow} \cdots \rightarrow \overline{2} \stackrel{1}{\rightarrow} \overline{1} \stackrel{0}{\rightarrow} 0 \stackrel{0}{\rightarrow} 1 \stackrel{1}{\rightarrow} 2 \cdots \stackrel{n-2}{\rightarrow} n-1 \stackrel{n-1}{\rightarrow} n \rightarrow \cdots$

The following proposition is an immediate consequence of the labelling of the crystals $B_{n}(\lambda)$ obtained in [12].

Proposition 5.2.2. Consider $\lambda \in \mathcal{P}_{m}$. The vertices of $B_{\infty}(\lambda)$ are indexed by the tableaux of $\mathbf{T}(\lambda)$.

Let $w$ be a word on $\mathcal{X}_{\infty}$. We have seen that there exists an integer $N$ such that for any integer $k \geq N, P_{k}(w)$ does not depend on $k$ and can be computed without the contraction relation (i.e. the number of boxes in $P(w)$ is equal to the length of $w)$. Thus it makes sense to consider the map

$$
P_{\infty}:\left\{\begin{array}{l}
\mathcal{X}_{\infty} \rightarrow \mathbf{T} \\
w \mapsto \lim _{n \rightarrow \infty} P_{n}(w) .
\end{array}\right.
$$

Set $G_{\infty}=\bigoplus_{l \geq 0} B_{\infty}(1)^{\otimes l}$. We define the relation $\sim$ by $w_{1} \sim w_{2}$ if and only if $w_{1}$ and $w_{2}$ appear at the same place in two isomorphic connected components of the crystal $G_{\infty}$.

Definition 5.2.3. The plactic monoids $P l\left(A_{\infty}\right), P l\left(B_{\infty}\right), P l\left(C_{\infty}\right)$ and $P l\left(D_{\infty}\right)$ are the quotients of the free monoids on the alphabets $\mathcal{X}_{A_{\infty}}, \mathcal{X}_{B_{\infty}}, \mathcal{X}_{C_{\infty}}$, and $\mathcal{X}_{D_{\infty}}$ respectively by the relations (26), (27), (28) and (29).

Proposition 5.2.4. Consider $w_{1}$ and $w_{2}$ in $\mathcal{X}_{\infty}^{*}$. We have the equivalences

$$
w_{1} \sim w_{2} \Longleftrightarrow w_{1} \equiv w_{2} \Longleftrightarrow P_{\infty}\left(w_{1}\right)=P_{\infty}\left(w_{2}\right) .
$$

Proof. By [15] and [16] we have for any integer $n \geq 1$ the equivalences

$$
w_{1} \sim_{n} w_{2} \Longleftrightarrow w_{1} \equiv_{n} w_{2} \Longleftrightarrow P_{n}\left(w_{1}\right)=P_{n}\left(w_{2}\right) .
$$

For $n$ sufficiently large we have seen that $P_{n}\left(w_{1}\right)=P_{\infty}\left(w_{1}\right)$ and $P_{n}\left(w_{2}\right)=P_{\infty}\left(w_{2}\right)$. Moreover there is no contraction during the computation of $P_{n}\left(w_{1}\right)$ and $P_{n}\left(w_{2}\right)$. 
Thus, for such an $n$, we have $w_{1} \equiv_{n} w_{2} \Longleftrightarrow w_{1} \equiv w_{2}$. This yields the equivalence $w_{1} \equiv w_{2} \Longleftrightarrow P_{\infty}\left(w_{1}\right)=P_{\infty}\left(w_{2}\right)$. The equality $P_{\infty}\left(w_{1}\right)=P_{\infty}\left(w_{2}\right)$ means that for any integer $p \geq n$, the words $w_{1}$ and $w_{2}$ occur at the same place in two isomorphic connected components of $B_{p}(1)^{\otimes \ell}$, where $\ell$ is the length of the words $w_{1}$ and $w_{2}$. Each connected component $\Gamma$ of $B_{\infty}(1)^{\ell}$ can be realized as the union of the connected components $\Gamma_{p}$ of $B_{p}(1)^{\otimes \ell}, p \geq n$, obtained by deleting in $\Gamma$ the arrows of colors $i \notin I_{p}$ together with the vertices they connect. Thus we have $P_{\infty}\left(w_{1}\right)=P_{\infty}\left(w_{2}\right) \Longleftrightarrow w_{1} \sim w_{2}$.

By the above proposition the plactic classes are labelled by the tableaux of $\mathbf{T}$. In the sequel we will write $[w]$ for the plactic class of the word $w$. We have in particular $\mathrm{w}\left(P_{\infty}(w)\right) \in[w]$.

5.3. RSK-correspondences and bi-crystal structures. By Proposition 5.2.2, for any positive integer $k$, the vertices of $B_{\infty}(k)$ are labelled by the row tableaux of length $k$. The combinatorial description of these tableaux depends on the type considered. More precisely a row tableau $R$ of type $\Delta_{\infty}(\Delta=A, B, C, D)$ and length $k$ is a row Young diagram of length $k$ filled from left to right by weakly increasing letters of $\mathcal{X}_{\Delta_{\infty}}$ and containing at most one letter 0 when $\Delta_{\infty}=B_{\infty}$. This implies that there are two kinds of rows of type $B_{\infty}$, namely the ones that contain the letters 0 , and the ones that do not. Similarly, since the letters 1 and $\overline{1}$ are not comparable in $D_{\infty}$, there are also two kinds of rows of type $D_{\infty}$ depending on whether they contain at least a letter 1 or not. According to the convention for the reading of a tableau, the reading of the row $R$ of length $r$ can be written $\mathrm{w}(R)=x_{1} \cdots x_{r}$ where $x_{1} \geq \cdots \geq x_{r}$.

Let $k$ be a nonnegative integer and consider $\tau=\left(\tau_{1}, \ldots, \tau_{k}\right) \in \mathbb{N}^{k}$. Set $B_{\infty}^{\tau}=$ $B_{\infty}\left(\tau_{1}\right) \otimes \cdots \otimes B_{\infty}\left(\tau_{k}\right)$ and

$$
\mathcal{W}_{\infty}=\bigoplus_{k=0}^{\infty} \bigoplus_{\tau \in \mathbb{N}^{k}} B_{\infty}^{\tau}
$$

Similarly for any integer $n \geq 1$, we define $B_{n}^{\tau}=B_{n}\left(\tau_{1}\right) \otimes \cdots \otimes B_{n}\left(\tau_{k}\right)$ and

$$
\mathcal{W}_{n}=\bigoplus_{k=0}^{\infty} \bigoplus_{\tau \in \mathbb{N}^{k}} B_{n}^{\tau}
$$

Let $\mathcal{Y}_{\infty}=\left\{y_{1}<y_{2}<\cdots\right\}$ be a totally ordered alphabet labelled by the positive integers. A bi-word on $\left(\mathcal{X}_{\infty}, \mathcal{Y}_{\infty}\right)$ is a word in the commutative variables $\left(\begin{array}{l}y_{j} \\ x_{i}\end{array}\right)$ where $x_{i} \in \mathcal{X}_{\infty}$ and $y_{j} \in \mathcal{Y}_{\infty}$. For types $A_{\infty}, B_{\infty}$ and $C_{\infty}$ each biword $W$ can be uniquely written in the form

$$
W=\left(\begin{array}{ccccccccccccccc}
y_{1} & \cdot & \cdot & y_{1} & y_{2} & \cdot & \cdot & y_{2} & \cdot & \cdot & \cdot & y_{k} & \cdot & \cdot & y_{k} \\
b_{1} & \cdot & \cdot & e_{1} & b_{2} & \cdot & \cdot & e_{2} & \cdot & \cdot & \cdot & b_{k} & \cdot & \cdot & e_{k}
\end{array}\right)=\left(\begin{array}{c}
w_{y} \\
w_{x}
\end{array}\right)
$$

by ordering first the letters $y_{j}$ and next the letters $x_{i}$ so that for any $p \in\{1, \ldots, k\}$, $b_{p} \cdots e_{p}$ is a word on $\mathcal{X}_{\infty}$ verifying $b_{p} \geq \cdots \geq e_{p}$. For type $D_{\infty}$, we can obtain similarly a canonical form for the biwords by requiring that the letters $\overline{1}$ appear on the left of the letters 1 in each word $b_{p} \cdots e_{p}$. Now consider a vertex $b \in \mathcal{W}_{\infty}$. There exists an integer $k$ and a $k$-tuple $\tau=\left(\tau_{1}, \ldots, \tau_{k}\right)$ such that $b \in B_{\infty}^{\tau}=B_{\infty}\left(\tau_{1}\right) \otimes \cdots \otimes B_{\infty}\left(\tau_{k}\right)$. Write $b=R_{1} \otimes \cdots \otimes R_{k}$ with $R_{p} \in B_{\infty}\left(\tau_{p}\right)$ for any $p \in\{1, \ldots, k\}$. In the sequel we will identify $b$ with the biword (30), where for any $p=1, \ldots, k, b_{p} \cdots e_{p}$ is the reading of the row $R_{p}$. For types $A_{\infty}$ and $C_{\infty}$, each biword can be interpreted as a 
vertex of $\mathcal{W}_{\infty}$ by the above identification. This is not true for types $B_{\infty}$ and $D_{\infty}$ due to the restrictive conditions on rows.

Consider $b \in B_{\infty}^{\tau}$. By abuse of notation, we write $P_{\infty}(b)$ for $P_{\infty}\left(w_{x}\right)$. For any $p \in\{1, \ldots, k\}$ write $Q^{(p)}$ for the shape of the tableau $P_{\infty}\left(b_{1} \cdots e_{p}\right)$. The shape $Q^{(p+1)}$ is obtained by adding $\tau_{p+1}$ boxes to $Q^{(p)}$; thus we can consider that $Q^{(p)} \subset Q^{(p+1)}$. Denote by $Q_{\infty}(b)$ the tableau obtained by filling $Q^{(1)}$ with letters $y_{1}$, and next filling each $Q^{(p+1)} \backslash Q^{(p)}$ with letters $y_{p+1}$.

Lemma 5.3.1. $Q_{\infty}(b)$ is a semistandard tableau on $\mathcal{Y}_{\infty}$.

Proof. By induction on $k$, it suffices to prove that the insertion of the row $R$ in a tableau $T$ gives a tableau $T^{\prime}$ such that $T^{\prime} \backslash T$ is a horizontal strip skew tableau (that is, has no boxes in the same column). There exists an integer $n$ such that $R, T$ and $T^{\prime}$ are tableaux of $\mathbf{T}_{n}$ verifying $T^{\prime}=P_{n}(\mathrm{w}(T) \mathrm{w}(R))$. Let $\lambda$ be the shape of $T$ and $r$ the length of $R$. Write $B(\mathrm{w}(T) \otimes \mathrm{w}(R))$ for the connected component of $B_{n}(\lambda) \otimes B\left(1^{r}\right)$ containing $\mathrm{w}(T) \otimes \mathrm{w}(R)$. The shape $\nu$ of $T^{\prime}$ is given by the weight of the highest weight vertex in $B(\mathrm{w}(T) \otimes \mathrm{w}(R))$. By using Lemma 2.2.1 and the fact that $|\nu|=|\lambda|+r$, one easily obtains that $\nu / \lambda$ is a horizontal strip.

In the sequel we will denote by SST the set of semistandard tableaux on the alphabet $\mathcal{Y}_{\infty}$.

Remarks. (i): Given a word $w$, its $Q$-symbol depends on the type considered. For example if we take $b=1 \otimes \overline{1} \otimes 1 \otimes \overline{1} \in B^{C_{\infty}}(1,1,1,1) \cap B^{D_{\infty}}(1,1,1,1)$, we will have

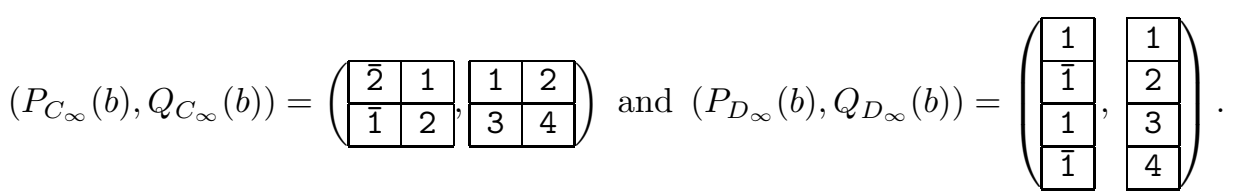

(ii): $Q_{\infty}(b)$ can also be considered as the sequence of the shapes of the tableaux $P_{\infty}\left(b_{1} \cdots e_{p}\right), p \in\{1, \ldots, k\}$.

(iii): It follows immediately from their definition that $P_{\infty}(b)$ and $Q_{\infty}(b)$ have the same shape.

Proposition 5.3.2. Consider a semistandard tableau $t$ of shape $\lambda$ and set $B_{\infty}(t)=$ $\left\{b \in \mathcal{W}_{\infty} \mid Q_{\infty}(b)=t\right\}$. Then $B_{\infty}(t)$ is a connected component of $\mathcal{W}_{\infty}$ isomorphic to $B_{\infty}(\lambda)$.

Proof. By the previous lemma, we know that $Q_{\infty}(b)$ is a semi-standard tableau. It follows from [15] and [16] that for any integer $n \geq 1, B_{n}(t)=B_{\infty}(t) \cap \mathcal{W}_{n}$ is a connected component isomorphic to $B_{n}(\lambda)$. Since $B_{\infty}(t)=\bigcup_{n \geq 1} B_{n}(t)$ and $B_{\infty}(\lambda)=\bigcup_{n \geq 1} B_{n}(\lambda)$ this gives the proposition.

For each $\Delta_{\infty} \in\left\{A_{\infty}, B_{\infty}, C_{\infty}, D_{\infty}\right\}$, set $\mathcal{E}_{\Delta_{\infty}}=\{(P, Q) \mid P$ and $Q$ are tableaux with the same shape, respectively of type $\Delta_{\infty}$ and semistandard on $\left.\mathcal{Y}_{\infty}\right\}$. The following theorem can be regarded as a generalization of the Robinson-SchenstedKnuth correspondence on biwords:

Theorem 5.3.3. The map

$$
\operatorname{RSK}:\left\{\begin{array}{l}
\mathcal{W}_{\infty} \rightarrow \mathcal{E}_{\infty} \\
b \mapsto\left(P_{\infty}(b), Q_{\infty}(b)\right)
\end{array}\right.
$$

is a one to one correspondence. 
Proof. Consider $b_{1}$ and $b_{2}$ in $\mathcal{W}_{\infty}$ such that $\operatorname{RSK}\left(b_{1}\right)=\operatorname{RSK}\left(b_{2}\right)$. Then by Propositions 5.2.4 and 5.3.2, $b_{1}$ and $b_{2}$ occur at the same place in the same connected component of $\mathcal{W}_{\infty}$. Hence $b_{1}=b_{2}$ and RSK is an injective map. Now consider $(P, Q) \in \mathcal{E}_{\infty}$. There exists an integer $n \geq 1$ such that $P \in \mathbf{T}_{n}$. Then by [15] and [16, there exists $b \in \mathcal{W}_{n} \subset \mathcal{W}_{\infty}$ such that $P_{n}(b)=P$ and $Q_{n}(b)=Q$. In this case we have $P_{\infty}(b)=P$ and $Q_{\infty}(b)=Q(b)$; thus RSK is a surjective map.

Remarks. (i): Let $b$ be a vertex of $B_{n}^{\tau}$ and denote by $Q_{n}(b)=\left(Q_{n}^{(1)}, \ldots, Q_{n}^{(k)}\right)$ the sequence of shapes of the tableaux $P_{n}\left(b_{1} \cdots e_{p}\right), p \in\{1, \ldots, k\}$. In general $Q_{n}^{(p+1)} \backslash Q_{n}^{(p)}$ is not a horizontal strip, for the number of boxes in $Q_{n}^{(p+1)}$ can be strictly less than that of $Q_{n}^{(p)}$. This implies in particular that the map $b \longmapsto\left(P_{C_{n}}(b), Q_{C_{n}}(b)\right)$ defined on $\mathcal{W}_{n}$ is not a one-to-one correspondence.

(ii): When any two consecutive shapes in $Q_{n}(b)$ differ by at most one box, one says that $Q_{n}(b)$ is an oscillating tableau. By [15] and [16], the map

$$
\mathrm{rs}_{n}:\left\{\begin{array}{l}
\bigoplus_{k=0}^{\infty} B_{n}\left(1^{k}\right) \rightarrow \mathcal{O}_{n} \\
b \mapsto\left(P_{n}(b), Q_{n}(b)\right)
\end{array}\right.
$$

where $\mathcal{O}_{\Delta_{n}}=\left\{(P, Q) \mid P\right.$ is a tableau and $Q$ an oscillating tableau of type $\left.\Delta_{n}\right\}$ is then a one-to-one correspondence. This difference with the situation described in (i) follows from the fact that the decomposition $V_{n}(\lambda) \otimes V_{n}\left(1^{k}\right)$ is not free multiplicity for types $B_{n}, C_{n}$ and $D_{n}$ when $k>1$.

(iii): The map

$$
\mathrm{rs}_{\infty}:\left\{\begin{array}{l}
\bigoplus_{k=0}^{\infty} B_{\infty}\left(1^{k}\right) \rightarrow \mathcal{O}_{\infty} \\
b \mapsto\left(P_{\infty}(b), Q_{\infty}(b)\right)
\end{array}\right.
$$

where $\mathcal{O}_{\Delta_{\infty}}=\left\{(P, Q) \mid P\right.$ and $Q$ are tableaux respectively of type $\Delta_{\infty}$ and standard on $\mathcal{Y}_{\infty}$ with the same shape $\}$ is also a one-to-one correspondence. Thus for any word $w$, the plactic class $[w]$ contains exactly $n_{\lambda}$ words (see Theorem 3.2.4) where $\lambda$ is the shape of $P(w)$. In particular, it is finite.

By Proposition 5.2.2, the set $\mathbf{T}(\lambda)$ of tableaux of type $\Delta_{\infty}=B_{\infty}, C_{\infty}, D_{\infty}$ can be endowed with the structure of a $U_{q}\left(\mathfrak{g}_{\Delta_{\infty}}\right)$-crystal so that for any two tableaux $T$ and $T^{\prime}$ in $\mathbf{T}(\lambda)$ and any Kashiwara operator $\widetilde{k}_{i}=\widetilde{e}_{i}, \widetilde{f}_{i}, i \geq 0$,

$$
\widetilde{k}_{i}(T)=T^{\prime} \Longleftrightarrow \widetilde{k}_{i}(\mathrm{w}(T))=\mathrm{w}\left(T^{\prime}\right) \text { in } B_{\Delta_{\infty}}(1)^{\otimes|\lambda|} .
$$

We proceed similarly to define a structure of a $U_{q}\left(\mathfrak{g}_{A_{\infty}}\right)$-crystal on the set $\mathbf{S S T}(\lambda)$ of semistandard tableaux of shape $\lambda$ on $\mathcal{Y}_{\infty}$. For any two tableaux $t$ and $t^{\prime}$ in $\operatorname{SST}(\lambda)$ and any Kashiwara operator $\widetilde{K}_{j}=\widetilde{E}_{j}, \widetilde{F}_{j}, j \geq 1$ we set

$$
\widetilde{K}_{j}(t)=t^{\prime} \Longleftrightarrow \widetilde{K}_{j}(\mathrm{w}(t))=\mathrm{w}\left(t^{\prime}\right) \text { in } B_{A_{\infty}}(1)^{\otimes|\lambda|} .
$$

By definition $\mathcal{W}_{\infty}$ is a $U_{q}\left(\mathfrak{g}_{\infty}\right)$-crystal. We can also endow it with the structure of a $U_{q}\left(\mathfrak{g}_{A_{\infty}}\right)$-crystal by setting

$$
\widetilde{K}_{j}(b)=\operatorname{RSK}^{-1}\left(P_{\infty}(b), \widetilde{K}_{j}\left(Q_{\infty}(b)\right)\right) .
$$


Theorem 5.3.4. $\mathcal{W}_{\infty}$ has the structure of a bi-crystal for the pair $\left(U_{q}\left(\mathfrak{g}_{\infty}\right)\right.$, $\left.U_{q}\left(\mathfrak{g}_{A_{\infty}}\right)\right)$; that is, for any Kashiwara operators $\widetilde{k}_{i}, i \in I$ and $\widetilde{K}_{j}, j \geq 1$ the following diagram commutes:

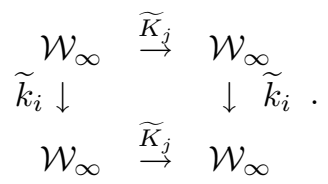

Proof. Consider $b \in \mathcal{W}_{\infty}$ and set $\operatorname{RSK}(b)=(P, Q)$. Then $\operatorname{RSK}\left(\widetilde{k}_{i}(b)\right)=\left(\widetilde{k}_{i}(P), Q\right)$, for $b$ and $\widetilde{k}_{i}(b)$ are in the same $U_{q}\left(\mathfrak{g}_{\infty}\right)$-connected component (see Proposition 5.3.2). This gives $\operatorname{RSK}\left(\widetilde{K}_{j}\left(\widetilde{k}_{i}(b)\right)\right)=\left(\widetilde{k}_{i}(P), \widetilde{K}_{j}(Q)\right)$. On the other hand, we have $\operatorname{RSK}\left(\widetilde{K}_{j}(b)\right)=\left(P, \widetilde{K}_{j}(Q)\right)$. Moreover $\operatorname{RSK}\left(\widetilde{k}_{i}\left(\widetilde{K}_{j}(b)\right)\right)=\left(\widetilde{k}_{i}(P), \widetilde{K}_{j}(Q)\right)$ since $\widetilde{K}_{j}(b)$ and $\widetilde{k}_{i}\left(\widetilde{K}_{j}(b)\right)$ are in the same $U_{q}\left(\mathfrak{g}_{\infty}\right)$-connected component. We obtain $\widetilde{K}_{j}\left(\widetilde{k}_{i}(b)\right)=\widetilde{k}_{i}\left(\widetilde{K}_{j}(b)\right)$ because the map RSK is a one-to-one correspondence

The RSK-type correspondences and the bi-crystal structures we have obtained in this paragraph can be referred to as the "symmetric case" by analogy to the situation in type $A$ where, for each $k \in \mathbb{N}$, the crystal $B_{\infty}(k)$ is that of the $k$-th symmetric power of the vector representation. It is also possible to define an RSKtype correspondence and a bi-crystal structure in the "anti-symmetric case", that is, when $\mathcal{W}_{\infty}$ is replaced by the crystal

$$
\widehat{\mathcal{W}}_{\infty}=\bigoplus_{k=0}^{\infty} \bigoplus_{\tau \in \mathbb{N}^{k}} \widehat{B}_{\infty}(\tau),
$$

where for any $\tau=\left(\tau_{1}, \ldots, \tau_{k}\right) \in \mathbb{N}^{k}, \widehat{B}_{\infty}(\tau)=B_{\infty}\left(1^{\tau_{1}}\right) \otimes \cdots \otimes B_{\infty}\left(1^{\tau_{k}}\right)$. By Proposition 5.2.2, the vertices of $B_{\infty}\left(1^{k}\right), k \in \mathbb{N}$ are labelled by the columns of height $k$ (see (20)). Consider $b=C_{1} \otimes \cdots \otimes C_{k} \in \widehat{B}_{\infty}(\tau)$. One associates to $b$ the tableau $\widehat{P}_{\infty}(b)=P_{\infty}\left(\mathrm{w}\left(C_{1}\right) \cdots \mathrm{w}\left(C_{k}\right)\right)$. Write $\left(Q_{n}^{(1)}, \ldots, Q_{n}^{(k)}\right)$ for the sequence of shapes of the tableaux $P_{\infty}\left(\mathrm{w}\left(C_{1}\right) \cdots \mathrm{w}\left(C_{p}\right)\right), p \in\{1, \ldots, k\}$. One proves that $Q_{n}^{(p+1)} \backslash Q_{n}^{(p)}$ is a vertical strip (that is, has no boxes in the same row). Let $\lambda$ be the shape of $\widehat{P}_{\infty}(b)$. Denote by $\widehat{Q}_{\infty}(b)$ the tableau obtained by filling $Q^{(1)}$ with letters $y_{1}$, and next filling each $Q^{(p+1)} \backslash Q^{(p)}$ with letters $y_{p+1}$. The rows of $\widehat{Q}_{\infty}(b)$ (read from left to right) strictly increase and its columns (read from top to bottom) weakly increase. This means that $\widehat{Q}_{\infty}(b)$ can be regarded as a semistandard tableau of shape $\lambda^{\prime}$, the conjugate partition of $\lambda$.

For each $\Delta_{\infty} \in\left\{A_{\infty}, B_{\infty}, C_{\infty}, D_{\infty}\right\}$, set $\widehat{\mathcal{E}}_{\Delta_{\infty}}=\{(P, Q) \mid P$ and $Q$ are tableaux with conjugate shapes, respectively of type $\Delta_{\infty}$ and semistandard on $\left.\mathcal{Y}_{\infty}\right\}$. By proceeding as for Theorem 5.3.3 we obtain:

Theorem 5.3.5. The map

$$
\widehat{\operatorname{RSK}}:\left\{\begin{array}{l}
\widehat{\mathcal{W}}_{\infty} \rightarrow \widehat{\mathcal{E}}_{\infty} \\
b \mapsto\left(\widehat{P}_{\infty}(b), \widehat{Q}_{\infty}(b)\right)
\end{array}\right.
$$

is a one to one correspondence.

This also permits us to endow $\widehat{\mathcal{W}}_{\infty}$ with a structure of a $U_{q}\left(\mathfrak{g}_{A_{\infty}}\right)$-crystal by setting

$$
\widetilde{K}_{j}(b)=\widehat{\operatorname{RSK}}^{-1}\left(P_{\infty}(b), \widetilde{K}_{j}\left(Q_{\infty}(b)\right)\right)
$$


for any Kashiwara operator $\widetilde{K}_{j}, j \geq 1$ associated to $U_{q}\left(\mathfrak{g}_{A_{\infty}}\right)$. Similarly to Theorem 5.3.4, we derive

Theorem 5.3.6. $\widehat{\mathcal{W}}_{\infty}$ has the structure of a bi-crystal for the pair $\left(U_{q}\left(\mathfrak{g}_{\infty}\right)\right.$, $\left.U_{q}\left(\mathfrak{g}_{A_{\infty}}\right)\right)$.

\section{A Plactic algebra For infinite Root systems}

6.1. Plactic Schur functions. Denote by $\mathbb{Z}\left[\mathcal{W}_{\infty}\right]$ the free $\mathbb{Z}$-algebra of basis $\mathcal{W}_{\infty}$, where the multiplication is defined by concatenation of tensors. More precisely, for any $\tau=\left(\tau_{1}, \ldots, \tau_{k}\right) \in \mathbb{N}^{k}, \tau=\left(\tau_{1}^{\prime}, \ldots, \tau_{k}^{\prime}\right) \in \mathbb{N}^{k^{\prime}}$ and $b=b_{1} \otimes \cdots \otimes b_{r} \in B_{\infty}^{\tau}, b^{\prime}=$ $b_{1}^{\prime} \otimes \cdots \otimes b_{r}^{\prime} \in B_{\infty}\left(\tau^{\prime}\right)$ we have

$$
b \cdot b^{\prime}=b_{1} \otimes \cdots \otimes b_{r} \otimes b_{1}^{\prime} \otimes \cdots \otimes b_{r}^{\prime} .
$$

Consider a partition $\lambda$ and $t$ a semistandard tableau of shape $\lambda$ on the alphabet $\mathcal{Y}_{\infty}$. We define the free Schur function associated to $t$ on $\mathbb{Z}\left[\mathcal{W}_{\infty}\right]$ by

$$
\mathbf{S}_{t}=\sum_{Q_{\infty}(b)=t} b=\sum_{b \in B_{\infty}(t)} b
$$

where $B_{\infty}(t)$ is the connected component of $\mathcal{W}_{\infty}$ associated to $t$ by Proposition 5.3.2. For any $b=b_{1} \otimes \cdots \otimes b_{r} \in B_{\infty}^{\tau}$, set $\mathrm{w}(b)=\mathrm{w}\left(b_{1}\right) \cdots \mathrm{w}\left(b_{r}\right)$. This is equivalent to saying that $\mathrm{w}(b)=w_{x}$ when $b$ is considered as the biword $\left(\begin{array}{l}w_{y} \\ w_{x}\end{array}\right)$. For each classical type $X \in\{A, B, C, D\}$, we define the plactic algebra $\mathbb{Z}[P l(\infty)]$ as the quotient of $\mathbb{Z}\left[\mathcal{W}_{\infty}\right]$ by the relations

$$
b=b^{\prime} \Longleftrightarrow \mathrm{w}(b) \equiv_{X} \mathrm{w}\left(b^{\prime}\right),
$$

where $\equiv_{X}$ is the plactic congruence corresponding to $X$. In particular $\mathbb{Z}[P l(\infty)]$ depends on the type considered. Denote by $\mathfrak{P}_{\infty}$ the canonical projection from $\mathbb{Z}\left[\mathcal{W}_{\infty}\right]$ to the plactic algebra $\mathbb{Z}[P l(\infty)]$. For any $b \in \mathcal{W}_{\infty}$, we thus have $\mathfrak{P}_{\infty}(b)=$ $P(\mathrm{w}(b))$. By Proposition 5.2.4, two vertices $b_{1}$ and $b_{2}$ in $\mathcal{W}_{\infty}$ occur at the same place in two isomorphic components if and only if $\mathfrak{P}_{\infty}\left(b_{1}\right)=\mathfrak{P}_{\infty}\left(b_{2}\right)$. In the sequel, we identify $T$ with the plactic class of its column reading $\mathrm{w}(T)$. Since the plactic classes are labelled by the tableaux, $\{T \mid T \in \mathbf{T}\}$ is a basis of $\mathbb{Z}[P l(\infty)]$. By Propositions 5.2 .4 and 5.3 .2 .

$$
\mathfrak{P}_{\infty}\left(\mathbf{S}_{t}\right)=\sum_{T \in B_{\infty}(\lambda)} T
$$

depends only of the shape $\lambda$. The right member of the above equality is called the plactic Schur function and is denoted by $\mathbf{S}_{\lambda}^{\infty}$.

Theorem 6.1.1. For each type $A_{\infty}, B_{\infty}, C_{\infty}, D_{\infty}$, the plactic Schur functions span a commutative subalgebra of $\mathbb{Z}[P l(\infty)]$. Moreover given two partitions $\lambda$ and $\mu$ we have

$$
\mathbf{S}_{\lambda}^{\infty} \cdot \mathbf{S}_{\mu}^{\infty}=\sum_{\nu \in \mathcal{P}} c_{\lambda, \mu}^{\nu} \mathbf{S}_{\nu}^{\infty}
$$

where $c_{\lambda, \mu}^{\nu}$ is a Littlewood-Richardson coefficient, thus does not depend on the type considered. 
Proof. In the plactic algebra $\mathbb{Z}[P l(\infty)]$, we have

$$
\begin{aligned}
\mathbf{S}_{\lambda}^{\infty} \cdot \mathbf{S}_{\mu}^{\infty} & =\sum_{T^{\prime} \in B_{\infty}(\lambda)} \sum_{T^{\prime \prime} \in B_{\infty}(\mu)} T^{\prime} \cdot T^{\prime \prime} \\
& =\sum_{T^{\prime} \otimes T^{\prime \prime} \in B_{\infty}(\lambda) \otimes B_{\infty}(\mu)} T \cdot T^{\prime \prime}=\sum_{\nu} \sum_{T \in B_{\infty}(\nu)} m(T) T,
\end{aligned}
$$

where $m(T)$ is the number of vertices $T^{\prime} \otimes T^{\prime \prime} \in B_{\infty}(\lambda) \otimes B_{\infty}(\mu)$ such that $T^{\prime} \cdot T^{\prime \prime}=T$ in $\mathbb{Z}[P l(\infty)]$. Let us write $\emptyset$ for the empty crystal. By assertion 2 of Corollary [3.2.5, Proposition 4.1.5 and Theorem 4.1.6, there is a crystal isomorphism

$$
\Upsilon_{\lambda, \mu}: B_{\infty}(\lambda) \otimes B_{\infty}(\mu) \stackrel{\simeq}{\rightarrow} \bigoplus_{\nu} \bigoplus_{r=1}^{c_{\lambda, \mu}^{\nu}} B_{\infty}(\nu) \otimes \emptyset^{\otimes r} .
$$

Since the plactic relations express crystal isomorphisms, for $T, T^{\prime}, T^{\prime \prime}$ respectively in $B_{\infty}(\nu), B_{\infty}(\lambda)$ and $B_{\infty}(\mu)$, we have the equivalences

$$
\begin{aligned}
T^{\prime} \cdot T^{\prime \prime}=T \Longleftrightarrow \mathrm{w}\left(T^{\prime}\right) \mathrm{w}\left(T^{\prime \prime}\right) & \equiv \mathrm{w}(T) \\
& \Longleftrightarrow \exists r \in\left\{1, \ldots, c_{\lambda, \mu}^{\nu}\right\} \mid \Upsilon_{\lambda, \mu}\left(T^{\prime} \otimes T^{\prime \prime}\right)=T \otimes \emptyset^{\otimes r} .
\end{aligned}
$$

This means that $m(T)=c_{\lambda, \mu}^{n}$ for any $T \in B_{\infty}(\nu)$. Hence we obtain

$$
\mathbf{S}_{\lambda}^{\infty} \cdot \mathbf{S}_{\mu}^{\infty}=\sum_{\nu} c_{\lambda, \mu}^{\nu} \sum_{T \in B_{\infty}(\nu)} T=\sum_{\nu} c_{\lambda, \mu}^{\nu} \mathbf{S}_{\nu}^{\infty}
$$

We deduce immediately the following corollary:

Corollary 6.1.2. The four algebras generated by the plactic Schur functions of types $A_{\infty}, B_{\infty}, C_{\infty}, D_{\infty}$ are all isomorphic to the algebra of symmetric functions.

6.2. Cauchy-type identities. Let $\mathcal{A}[[X, Y]]$ be the $\mathbb{Z}$-algebra of formal series in the noncommutative variables $x_{i} \in \mathcal{X}_{\infty}$ and $y_{j} \in \mathcal{Y}_{\infty}$ subject to the relations

$$
\left\{\begin{array}{l}
x_{\bar{i}} x_{i}=x_{i+1} x_{\overline{i+1}} \text { for any } i \geq 1, x_{i^{\prime}} x_{i}=x_{i} x_{i^{\prime}} \text { for any }\left(i, i^{\prime}\right) \text { with } i^{\prime} \neq-i \\
y_{j} y_{j^{\prime}}=y_{j^{\prime}} y_{j} \text { for any }\left(j, j^{\prime}\right) \text { and } x_{i} y_{j}=y_{j} x_{i} \text { for any }(i, j) .
\end{array}\right.
$$

By using relations (31), each monomial in the variables $x_{i}$ can be uniquely written in the form

$$
x^{\alpha}=x_{n}^{\alpha_{n}} \cdots x_{1}^{\alpha_{1}} x_{0}^{\alpha_{0}} x_{\overline{1}}^{\alpha_{\bar{T}}} \cdots x_{\bar{m}}^{\alpha_{\pi}},
$$

where the $\alpha_{i}$ 's are nonnegative integers such that $\alpha_{n} \alpha_{\bar{m}} \neq 0$.

To each word $w=x_{1} \cdots x_{\ell}$ on $\mathcal{X}_{\infty}$, we associated the monomial $x^{w}=x_{1} \cdots x_{\ell} \in$ $\mathcal{A}[[X]]$. By (31),

$$
w_{1} \equiv w_{2} \Longrightarrow x^{w_{1}}=x^{w_{2}}
$$

for $w_{1}$ and $w_{2}$ two words on $\mathcal{X}_{\infty}$.

Given $x \in \mathcal{X}_{\infty}$ and $y \in \mathcal{Y}_{\infty}$ we have $\frac{1}{1-x y} \in \mathcal{A}[[X, Y]]$. Moreover in $\mathcal{A}[[X, Y]]$ we can write

$$
\begin{aligned}
& \frac{1}{1-x_{\bar{i}} y} \frac{1}{1-x_{i} y^{\prime}}=\frac{1}{1-x_{i+1} y} \frac{1}{1-x_{\overline{i+1}} y^{\prime}} \text { for any } i \geq 1 \\
& \frac{1}{1-x_{i} y} \frac{1}{1-x_{j} y^{\prime}}=\frac{1}{1-x_{j} y^{\prime}} \frac{1}{1-x_{i} y} \text { if } j \neq-i \text { or } i=j=0 .
\end{aligned}
$$


For any partition $\lambda$, denote by $s_{\lambda}(Y)$ the infinite Schur function of type $A$ in the variables $y \in \mathcal{Y}_{\infty}$. For $\Delta=A, B, C, D$, set

$$
S_{\lambda}^{\Delta_{\infty}}(X)=\sum_{T \in \mathbf{T}(\lambda)} x^{\mathrm{w}(T)} \in \mathcal{A}[[X]] .
$$

Remarks. (i) : Note that for types $B_{\infty}, C_{\infty}$ and $D_{\infty}$, it is impossible to specialize $x_{\bar{i}}=\frac{1}{x_{i}}$ in $S_{\lambda}^{\Delta_{\infty}}(X)$ because $\mathbf{T}(\lambda)$ contains an infinite number of tableaux with the same weight. Thus $S_{\lambda}^{\Delta_{\infty}}(X)$ cannot be interpreted as an ordinary character for classical Lie algebras.

(ii) : Similarly, one cannot specialize the letters of a tableau $T \in \mathbb{Z}[P l(\infty)]$ in commutative variables for types $B_{\infty}, C_{\infty}$ and $D_{\infty}$. Indeed, if we do so, the result could depend on the representative chosen in the plactic class $T$ since words which are plactic equivalent will give distinct monomials in general. This is because the plactic relations are not homogeneous for types $B_{\infty}, C_{\infty}$ and $D_{\infty}$. This implies notably that the plactic Schur functions we have defined cannot be related to the universal characters of Koike and Terada in a simple way.

For each type $\Delta_{\infty}(\Delta=A, B, C, D)$ set

$$
K_{\Delta_{\infty}}(X, Y)=\left\{\begin{array}{l}
\prod_{i=1}^{\infty} \prod_{j=1}^{\infty} \frac{1}{1-x_{i} y_{j}} \text { if } \Delta=A, \\
\prod_{i=1}^{\infty} \prod_{j=1}^{\infty} \frac{1+x_{0}}{\left(1-x_{i} y_{j}\right)\left(1-x_{\bar{\imath}} y_{j}\right)} \text { if } \Delta=B, \\
\prod_{i=1}^{\infty} \prod_{j=1}^{\infty} \frac{1}{\left(1-x_{i} y_{j}\right)\left(1-x_{\bar{\imath}} y_{j}\right)} \text { if } \Delta=C, \\
\prod_{i=2}^{\infty} \prod_{j=1}^{\infty} \frac{1}{\left(1-x_{i} y_{j}\right)\left(1-x_{\bar{\imath}} y_{j}\right)}\left(\frac{1}{1-x_{1} y_{j}}+\frac{1}{1-x_{\top} y_{j}}\right) \text { if } \Delta=D .
\end{array}\right.
$$

Theorem 6.2.1. For $\Delta=A, B, C, D$, we have the following Cauchy-type identities in $\mathcal{A}[[X, Y]]$ :

$$
K_{\Delta_{\infty}}(X, Y)=\sum_{\lambda \in \mathcal{P}} S_{\lambda}^{\Delta_{\infty}}(X) s_{\lambda}(Y) .
$$

Proof. To each biword $W=\left(\begin{array}{c}w_{y} \\ w_{r}\end{array}\right) \in \mathcal{W}_{\infty}$ (see (30) $)$ such that $w_{x}=x_{i_{1}} \cdots x_{i_{r}}$ and $w_{y}=y_{i_{1}} \cdots y_{i_{r}}$ we associate the monomial $x^{w_{x}} y^{w_{y}}=x_{i_{1}} \cdots x_{i_{r}} y_{i_{1}} \cdots y_{i_{r}} \in \mathcal{A}[[X, Y]]$. From the description of the row tableaux given in $\$ 5.3$ and by using relations (31), we obtain in $\mathcal{A}[[X, Y]]$,

$$
K_{\Delta_{\infty}}(X, Y)=\sum_{W \in \mathcal{W}_{\infty}} x^{w_{x}} y^{w_{y}} .
$$

By Theorem 5.3.3 the map RSK is a one-to-one correspondence between the biwords $W \in \mathcal{W}_{\infty}$ and the pair $(T, t)$ where $T$ and $t$ are respectively a tableau on $\mathcal{X}_{\infty}$ and a semistandard tableau on $\mathcal{Y}$ with the same shape. Moreover we have $x^{w_{x}}=x^{\mathrm{w}(T)}$ and $y^{w_{y}}=y^{\mathrm{w}(t)}$ (see (32)) in $\mathcal{A}[[X, Y]]$. This gives

$$
\begin{aligned}
K_{\Delta_{\infty}}(X, Y) & =\sum_{\lambda} \sum_{(T, t) \in \mathbf{T}(\lambda) \times \mathbf{S S T}(\lambda)} x^{\mathrm{w}(T)} y^{\mathrm{w}(t)} \\
& =\sum_{\lambda} \sum_{T \in \mathbf{T}(\lambda)} x^{\mathrm{w}(T)} \sum_{t \in \mathbf{S S T}(\lambda)} y^{\mathrm{w}(t)}=\sum_{\lambda \in \mathcal{P}} S_{\lambda}^{\Delta_{\infty}}(X) s_{\lambda}(Y) .
\end{aligned}
$$

Remarks. (i): It is possible to derive Cauchy identities for a finite alphabet $\mathcal{Y}_{n}=$ $\left\{y_{1}<\cdots<y_{n}\right\}$ by specializing the variables $y_{i}, i>n$ to 0 in (34). 
(ii): Such a specialization is not possible for the variables $x_{i}$ due to the relations $x_{\bar{\imath}} x_{i}=x_{i+1} x_{\overline{i+1}}$. This means that, except for type $A$, Cauchy-type identities (34) only make sense in the infinite rank case. This is related to the fact that the Robinson-Schensted correspondence of Theorem 5.3.3 cannot be restricted to types $B_{n}, C_{n}$ and $D_{n}$ (see Remark (iii) after Theorem [5.3.3).

(iii): The previous arguments show that it is not possible to recover the Littlewood formulas 19 from (34). Note that a combinatorial proof of Littlewood's identity for type $C_{n}$ based on an RSK-type correspondence have been obtained by Sundaram 23. As far as the author is aware, this correspondence, which uses the Berele insertion algorithm, is not compatible with Kashiwara crystal basis theory.

\section{COMBinatorial QUESTION}

The bi-crystal structures we have obtained in Theorems 5.3.4 and 5.3.5 are defined implicitly by using the RSK-type correspondences on $\mathcal{W}_{\infty}$ and $\widehat{\mathcal{W}}_{\infty}$. By using the results obtained in [2], one can easily deduce that the $\left(U_{q}\left(\mathfrak{g}_{A_{\infty}}\right), U_{q}\left(\mathfrak{g}_{A_{\infty}}\right)\right)$ bi-crystals (that is, corresponding to the case $\Delta=A$ ) can be naturally labelled by certain infinite arrays making the symmetry between the two crystal structures apparent. It would be interesting to define analogues of these arrays for the $\left(U_{q}\left(\mathfrak{g}_{\infty}\right), U_{q}\left(\mathfrak{g}_{A_{\infty}}\right)\right)$ bi-crystals of Theorems 5.3.4 and 5.3.5 when $\mathfrak{g}_{\infty} \neq \mathfrak{g}_{A_{\infty}}$.

\section{REFERENCES}

[1] A. Berele, A Schensted-type correspondence for the symplectic group, J. Combin. Theory (A), 43, 320-328 (1986). MR867655 (88b:20027)

[2] V. I. Danilov, G. A. Koshevoy, Bi-crystal and $(G L(V), G L(W))$-duality, RIMS no. 1458, 2004.

[3] W. Fulton, Young tableaux, London Mathematical Society, Student Text 35, 1997. MR.1464693 (99f:05119)

[4] J. C. Jantzen, Lectures on quantum groups, Graduate Studies in Math. 6, A.M.S., 1995. MR1359532 (96m:17029)

[5] N. Jing, K. C. MisRA, M. OKADO, q-Wedge modules for quantized enveloping algebras of classical type, Journal of Algebra, 230 (2000), 518-539. MR.1775802 (2001i:17014)

[6] A. M. HAmel, Deterninantal forms for symplectic and orthogonal Schur functions, Can. J. Math. 49 (2), 263-282 (1997). MR1447491 (98e:05107)

[7] V. G. KAC, Bombay Lectures on highest weight representations of infinite dimensional Lie algebras, Advanced Series in Mathematical Physics Vol. 2, 1988.

[8] J. Hong, S. J. KANG, Introduction to quantum groups and crystals bases, A.M.S., 2002, GSM/12. MR:1881971 (2002m:17012)

[9] V. G. KaC, Infinite Dimensional Lie Algebras, third ed., Cambridge University Press, (1990). MR.1104219 (92k:17038)

[10] M. KASHIWARA, On crystal bases of the q-analogue of universal enveloping algebras, Duke Math. J, 63 (1991), 465-516. MR1115118 (93b:17045)

[11] M. Kashiwara, On crystal bases, Canadian Mathematical Society, Conference Proceedings, 16 (1995), 155-197. MR1357199 (97a:17016)

[12] M. Kashiwara, T. NAKASHima, Crystal graphs for representations of the q-analogue of classical Lie algebras, Journal of Algebra 165 (1994), 295-345. MR.1273277 (95c:17025)

[13] A. Lascoux, Double crystal graphs, Studies in the memory of Issai Schur, Progess in Math., 210, Birkhaüser, 95-114 (2003). MR.1985724 (2004f:05186)

[14] A. LAsCoux, Symmetric functions and combinatorial operators on polynomials, CBMS, number 99 (2003). MR2017492(2005b:05217)

[15] C. Lecouvey, Schensted-type correspondence, Plactic Monoid and Jeu de Taquin for type $C_{n}$, Journal of Algebra, 247, 295-331 (2002). MR.1877856 (2003e:05144)

[16] C. Lecouvey, Schensted-type correspondences and plactic monoids for types $B_{n}$ and $D_{n}$, Journal of Algebraic Combinatorics, vol 18 no. 2, 99-133 (2003). MR2002620(2004g:05153) 
[17] C. Lecouvey, An algorithm for computing the global basis of a finite dimensional irreducible $U_{q}\left(s o_{2 n+1}\right)$ or $U_{q}\left(s o_{2 n}\right)$-module, Communications in Algebra, vol 32, no. 5, 1969-1996 (2204). MR2099716 (2006d:17013)

[18] P. Littelmann, A plactic algebra for semisimple Lie algebras, Adv. in Math. 124, 312-331 (1996). MR:1424313 (98c:17009)

[19] D. E. LitTLEWOOD, The theory of group characters and matrix representations of groups, Oxford University Press, second edition (1958). MR0002127 (2:3a)

[20] M. Lothaire, Algebraic combinatorics of words, Encyclopedia of Mathematics and its applications, Cambridge University Press, 90, 164-196. MR1905123 (2003i:68115)

[21] S. OKadA, A Robinson-Schensted-type algorithm for $\mathrm{SO}(2 n, \mathbb{C})$, J. Algebra 143, 334-372 (1991). MR 1132576 (92k:05133)

[22] R. Proctor, A Schensted algorithm which models tensor representations of the orthogonal group, Canad. J. Math. 42, 28-49 (1990). MR1043509 (91a:20045)

[23] S. Sundaram, The Cauchy identity for $\operatorname{Sp}(2 n)$, J. Combin. Theory, ser. A 53, 209-238 (1990). MR:1041446(91i:05122)

[24] S. Sundaram, Orthogonal tableaux and an insertion scheme for $\mathrm{SO}_{2 n+1}$, J. Combin. Theory, ser. A 53, 239-256 (1990). MR1041447 (91c:05199)

[25] M. Van Leeuwen, Double crystal of binary and integral matrices, arXiv:math/0605420.

Laboratoire de Mathématiques Pures et Appliquées, Joseph Liouville, B.P. 699, 62228

Calais Cedex, France 